\title{
Dislocated Soft Metric Space with Soft Fixed Point Theorems
}

\author{
Balaji Raghunath Wadkar1, Vishnu Narayan Mishra ${ }^{2,3 *}$, Ramakant Bhardwaj ${ }^{4}$, Basant Singh" \\ ${ }^{1}$ Department of Mathematics, AISECT University, Bhopal, India \\ ${ }^{2}$ Applied Mathematics \& Humanities Department, S.V. National Institute of Technology, Surat, India \\ ${ }^{3}$ Department of Mathematics, Indira Gandhi National Tribal University, Amarkantak, India \\ ${ }^{4}$ Department of Mathematics, TIT Group of Institutes, Bhopal, India \\ Email: wbrlatur@gmail.com, ^vishnunarayanmishra@gmail.com,rkbhardwaj100@gmail.com,brwlatur@gmail.com
}

How to cite this paper: Wadkar, B.R., Mishra, V.N., Bhardwaj, R. and Singh, B. (2017) Dislocated Soft Metric Space with Soft Fixed Point Theorems. Open Journal of Discrete Mathematics, 7, 108-133. https://doi.org/10.4236/ojdm.2017.73012

Received: September 21, 2016

Accepted: June 13, 2017

Published: June 16, 2017

Copyright (c) 2017 by authors and Scientific Research Publishing Inc. This work is licensed under the Creative Commons Attribution International License (CC BY 4.0).

http://creativecommons.org/licenses/by/4.0/ (c) (i) Open Access

\begin{abstract}
In the present paper, we define Dislocated Soft Metric Space and discuss about the existence and uniqueness of soft fixed point of a cyclic mapping in soft dislocated metric space. We also prove the unique soft fixed point theorems of a cyclic mapping in the context of dislocated soft metric space. Examples are given for support of the results.
\end{abstract}

\section{Keywords}

Soft Set, Soft Fixed Point, Soft Point of Soft Mapping, Soft Metric Space, Dislocated Soft Metric Space

\section{Introduction}

The soft set theory is one of the branches of mathematics, which aims to describe phenomena and concepts of an ambiguous, undefined vague and imprecise meaning, which was initiated by Molodtsov [1]. This theory is applicable where there are no clearly defined mathematical models. Recently many papers concerning soft sets have been published (see [2]-[8]). In many aspects of Mathematics, fixed point theory has wonderful applications. Shabir and Naz [9] presented soft topological spaces and they investigated some properties of soft topological spaces. Later many researchers were studied about soft topological spaces. In these studies, concepts of soft fixed point are expressed by different approaches. Das and Samanta [10] [11] introduced a different notion of soft metric space by using different concept of soft point and investigated some basic properties of these. In 2000, Hitzler and Seda [12] introduced the notion of dislocated metric space, in which self distance of a point need not be equal to zero. Aage and Salunke [13] established some important fixed point theorem in single 
and pair of mappings in dislocated metric space. Later Karapnar and Salimi [14] discussed the existence and uniqueness of fixed point of a cyclic mapping in the context of metric like space. The study of common fixed point of mapping in dislocated metric space satisfying certain contractive condition has been at the Centre of vigorous research activity (see [15] [16] [17] [18]). Dislocated metric space plays very important role in topology, logical programming and in electronic engineering. Recently Wadkar et al. [19], Mishra et al. [20] [21] [22] [23], Deepmala and pathak [24], Wadkar et al. [25], [26] discussed and proved fixed point theorems by employing different concepts.

In the present paper, we discuss about the investigations concerning the existence and uniqueness of soft fixed point of a cyclic mapping in soft dislocated metric space. We also prove the unique soft fixed point theorems of a cyclic mapping in the context of dislocated metric space. To check the validity of the result we give the examples. Before starting to prove main result, some basic definitions are required.

Definition 1.1: Let $X$ and $E$ are respectively an initial inverse set and a parameter set. A soft set over $X$ is pair denoted by $(Y, E)$ if and only if $Y$ is a mapping from $E$ into the set of all subsets of the set $X$, i.e. $Y: E \rightarrow P(x)$, where $P(x)$ is the power set of $X$.

Example of Soft Set: Let $(\breve{C}, \breve{D})$ be soft set, which describes the Nature of workers at Industry. Suppose that $U=\left\{u_{1}, u_{2}, u_{3}, u_{4}\right\}$, i.e. universe of four workers. Let a set of decision parameters be $\breve{D}=\left\{s_{1}, s_{2}, s_{3}, s_{4}, s_{5}\right\}$. Now

$s_{i}(i=1,2,3,4,5)$ stand for the parameters: high working speed, slow working speed, average working speed, work delay working speed and no working speed properly respectively.

Now consider $\tilde{C}\left(s_{1}\right)=\left\{u_{1}, u_{3}\right\}, \tilde{C}\left(s_{2}\right)=\left\{u_{1}, u_{3}, u_{4}\right\}, \tilde{C}\left(s_{3}\right)=U$, $\tilde{C}\left(s_{4}\right)=\left\{u_{2}, u_{4}\right\}$ and $\tilde{C}\left(s_{5}\right)=$ empty. By consisting of the following collection of approximations, the soft set $(\breve{C}, \breve{D})$ can be viewed.

$(\tilde{C}, \tilde{D})=\left\{\left(\right.\right.$ high working speed, $\left.\left\{u_{1}, u_{3}\right\}\right),\left(\right.$ slow working speed, $\left.\left\{u_{1}, u_{3}, u_{4}\right\}\right)$, (average working speed, $U$ ), (work delay working speed, $\left.\left\{u_{2}, u_{4}\right\}\right)$, (no working speed, empty)\}.

Definition 1.2: The intersection of two soft sets $(Y, A)$ and $(Z, B)$ over $X$ is a soft set over $X$ denoted by $(I, C)$ and is given by $(Y, A) \tilde{\cap}(Z, B)=(I, C)$, where $C=A \cap B$ and $\forall \varepsilon \in C, I(\varepsilon)=Y(\varepsilon) \cap Z(\varepsilon)$.

Definition 1.3: The union of two soft sets $(Y, A)$ and $(Z, B)$ over $X$ is the soft set $(I, C)$, where $C=A \cup B$ and for all $k$ in $C$,

$$
I(k)=\left\{\begin{array}{l}
Y(k), \text { if } k \text { is an element of } A-B, \\
Z(k), \text { if } k \text { is an element of } B-A, \\
Y(k) \cup Z(k), \text { if } k \text { is an element of } A \cap B .
\end{array}\right.
$$

This relationship is denoted by $(Y, A) \tilde{\cup}(Z, B)=(I, C)$.

Definition 1.4: A soft set $(Y, A)$ over $X$ is said to be a null soft set if $Y(k)=$ empty, for all $k$ in $A$ and is denoted by $\Phi$. 
Definition 1.5: For all $k \in A$, if $Y(k)=X$ then $(Y, A)$ is called an absolute soft set over $X$.

Definition 1.6: The difference of two soft sets $(F, E)$ and $(G, E)$ over $X$ is a soft set $(H, E)$ over $X$, denoted by $(F, E) \backslash(G, E)$ and is defined as $H(x)=F(x) \backslash G(x), \forall x \in E$.

Definition 1.7: The complement of soft set $(Y, A)$ is denoted by $(Y, A)^{c}$ and is defined as $(Y, A)^{c}=\left(Y^{c}, A\right)$, where $Y^{c}: A \rightarrow P(X)$ is a mapping given by $Y^{c}(\beta)=X-Y(\beta)$, for all $\beta$.

Definition 1.8: Let $B(R)$ be the collection of all non-empty bounded subsets of $R$ and $E$ taken as a set of parameters. Then the mapping $Y: E \rightarrow B(R)$ is called a soft real set. It is denoted by $(Y, E)$.

Definition 1.9: For two soft real numbers $\tilde{m}$ and $\tilde{n}$ the following conditions holds:

(i) $\tilde{m} \leq \tilde{n}$ if $\tilde{m}(s) \leq \tilde{n}(s)$, for all $s \in E$;

(ii) $\tilde{m} \geq \tilde{n}$ if $\tilde{m}(s) \geq \tilde{n}(s)$, for all $s \in E$;

(iii) $\tilde{m}<\tilde{n}$ if $\tilde{m}(s)<\tilde{n}(s)$, for all $s \in E$;

(iv) $\tilde{m}>\tilde{n}$ if $\tilde{m}(s)>\tilde{n}(s)$, for all $s \in E$.

Definition 1.10: A soft set $(P, E)$ over $X$ is said to have a soft point if there is exactly one $s \in E$ such that $P(s)=\{x\}$, for some $x \in X$, also $P\left(s^{\prime}\right)=\varphi$, $\forall s^{\prime} \in E /\{s\}$. It will be denoted by $\tilde{x}_{s}$.

Definition 1.11: Two soft points $\tilde{x}_{i}, \tilde{y}_{j}$ are said to be equal if $i=j$ and $P(i)=P(j)$ i.e. $x=y$. Hence $\tilde{x}_{i} \neq \tilde{y}_{j} \Leftrightarrow x \neq y$ or $i \neq j$.

Definition 1.12: A mapping $\tilde{\rho}: S P(\tilde{X}) \times S P(\tilde{X}) \rightarrow R(E) *$ is soft metric on soft set $\tilde{X}$ with following properties.

SM1. for all $\tilde{x}_{s_{1}}, \tilde{y}_{s_{2}} \in \tilde{X}, \quad \tilde{\rho}\left(\tilde{x}_{s_{1}}, \tilde{y}_{s_{2}}\right) \tilde{\geq} \overline{0}$;

SM2. $\tilde{\rho}\left(\tilde{x}_{s_{1}}, \tilde{y}_{s_{2}}\right)=\overline{0}$, if and only if $\tilde{x}_{s_{1}}=\tilde{y}_{s_{2}}$;

SM3. for all $\tilde{x}_{s_{1}}, \tilde{y}_{s_{2}} \in \tilde{X}, \quad \tilde{\rho}\left(\tilde{x}_{s_{1}}, \tilde{y}_{s_{2}}\right)=\tilde{\rho}\left(\tilde{y}_{s_{2}}, \tilde{x}_{s_{1}}\right)$;

SM4. for all $\tilde{x}_{s_{1}}, \tilde{y}_{s_{2}}, \tilde{z}_{s_{3}} \in \tilde{X}, \quad \tilde{\rho}\left(\tilde{x}_{s_{1}}, \tilde{z}_{s_{3}}\right) \leq \tilde{\rho}\left(\tilde{x}_{s_{1}}, \tilde{y}_{s_{2}}\right)+\tilde{\rho}\left(\tilde{y}_{s_{2}}, \tilde{z}_{s_{3}}\right)$.

The soft set $\tilde{X}$ with a soft metric $\tilde{\rho}$ defined on $\tilde{X}$ is called a soft metric space and denoted by $(\tilde{X}, \tilde{\rho}, E)$.

Definition 1.13: Let us consider a soft metric $(\tilde{X}, \tilde{\rho}, E)$ and $\tilde{\alpha}$ be a non-negative soft real number. The soft open ball with center at $\tilde{X}_{s}$ and radius $\tilde{\alpha}$ is given by

$$
B\left(\tilde{x}_{s}, \tilde{\alpha}\right)=\left\{\tilde{y}_{s^{\prime}} \in \tilde{X}: \tilde{\rho}\left(\tilde{x}_{s}, \tilde{y}_{s^{\prime}}\right) \leq \tilde{\alpha}\right\} \subset S P(\tilde{X}),
$$

and the soft closed ball with center at $\tilde{X}_{s^{\prime}}$ and radius $\tilde{\alpha}$ is given by

$$
B\left(\tilde{x}_{s}, \tilde{\alpha}\right)=\left\{\tilde{x}_{s^{\prime}} \in \tilde{X}: \tilde{\rho}\left(\tilde{x}_{s^{\prime}}, \tilde{y}_{s^{\prime}}\right) \leq \tilde{\alpha}\right\} \subset S P(\tilde{X}) .
$$

Definition 1.14: A sequence $\left\{\tilde{x}_{\lambda_{n}}^{n}\right\}$ of soft points in soft metric space $(\tilde{X}, \tilde{\rho}, E)$ is said to be convergent in $(\tilde{X}, \tilde{\rho}, E)$ if there is a soft point $\tilde{y}_{\mu} \tilde{\in} \tilde{X}$ such that $\tilde{\rho}\left(\tilde{x}_{\lambda_{n}}^{n}, \tilde{y}_{\mu}\right) \rightarrow \tilde{0}$ as $n \rightarrow \infty$, that is for every $\tilde{\varepsilon}>\overline{0}$, there is a natural number $N=N(\tilde{\varepsilon})$ such that $\overline{0} \leq \tilde{\rho}\left(\tilde{x}_{\lambda_{n}}^{n}, \tilde{y}_{\mu}\right) \tilde{<} \tilde{\varepsilon}$, whenever $n>N$.

Definition 1.15: Let $(\tilde{X}, \tilde{\rho}, E)$ be a soft metric space, then the sequence 
$\left\{\tilde{X}_{\lambda_{n}}^{n}\right\}$ of soft points in $(\tilde{X}, \tilde{\rho}, E)$ is said to be a Cauchy sequence in $\tilde{X}$, if corresponding to every $\tilde{\varepsilon} \tilde{\geq} \overline{0}$, there exist $m \in N$ such that $\tilde{\rho}\left(\tilde{x}_{\lambda_{i}}^{i}, \tilde{y}_{\lambda_{j}}^{j}\right) \tilde{\leq} \tilde{\varepsilon}, \forall i, j \geq m$, i.e. $\tilde{\rho}\left(\tilde{x}_{\lambda_{i}}^{i}, \tilde{y}_{\lambda_{j}}^{j}\right) \rightarrow \overline{0}$ as $i, j \rightarrow \infty$.

Definition 1.16: The soft metric space $(\tilde{X}, \tilde{\rho}, E)$ is called complete, if every Cauchy sequence in $\tilde{X}$ converges to some point of $\tilde{X}$.

Definition 1.17: Let $(\tilde{X}, \tilde{\rho}, E)$ be a soft metric space. A function $(f, \varphi):(\tilde{X}, \tilde{\rho}, E) \rightarrow(\tilde{X}, \tilde{\rho}, E)$ is called a soft contraction mapping if there is a soft real number $\alpha \in R, \overline{0} \leq \alpha<\overline{1}$ such that for every point $\tilde{x}_{\lambda}, \tilde{y}_{\mu} \in \operatorname{SP}(X)$, we have

$$
\tilde{\rho}\left((f, \varphi)\left(\tilde{x}_{\lambda}\right),(f, \varphi)\left(\tilde{y}_{\mu}\right)\right) \leq \alpha \tilde{\rho}\left(\tilde{x}_{\lambda}, \tilde{y}_{\mu}\right) .
$$

Definition 1.18: A mapping $\tilde{\rho}: S P(\tilde{X}) \times S P(\tilde{X}) \rightarrow R(E)^{*}$ is said to be dislocated soft metric on the soft set $\tilde{X}$ if $\tilde{\rho}$ satisfies the following conditions:

(d1) $\tilde{\rho}\left(\tilde{x}_{s_{1}}, \tilde{y}_{s_{2}}\right)=\overline{0}$ then $\tilde{x}_{s_{1}}=\tilde{y}_{s_{2}}$,

(d2) $\tilde{\rho}\left(\tilde{x}_{s_{1}}, \tilde{y}_{s_{2}}\right)=\tilde{\rho}\left(\tilde{y}_{s_{2}}, \tilde{x}_{s_{1}}\right)$, for all $\tilde{x}_{s_{1}}, \tilde{y}_{s_{2}} \in \tilde{X}$,

(d3) $\tilde{\rho}\left(\tilde{x}_{s_{1}}, \tilde{z}_{s_{3}}\right) \leq \tilde{\rho}\left(\tilde{x}_{s_{1}}, \tilde{y}_{s_{2}}\right)+\tilde{\rho}\left(\tilde{y}_{s_{2}}, \tilde{z}_{s_{3}}\right)$, for all $\tilde{x}_{s_{1}}, \tilde{y}_{s_{2}}, \tilde{z}_{s_{3}} \in \tilde{X}$.

The soft set $\tilde{X}$ with soft dislocated metric $\tilde{\rho}$ defined on $\tilde{X}$ is called a dislocated soft metric space and denoted by $(\tilde{X}, \tilde{\rho}, E)$.

\section{Main Results}

Theorem 2.1: Let $A$ and $B$ be two non-empty closed subsets of a complete dislocated soft metric space $(\tilde{X}, \tilde{\rho}, E)$. Suppose $(f, \varphi): A \cup B \rightarrow A \cup B$ is cyclic and satisfy the following:

(c) There exist a constant $k \in(0,1)$ such that

$\tilde{\rho}\left((f, \varphi) x_{\lambda},(f, \varphi) y_{\mu}\right) \leq k \tilde{\rho}\left(x_{\lambda}, y_{\mu}\right)$ for all $x_{\lambda} \in A, \quad y_{\mu} \in B$.

Then $(f, \varphi)$ has a unique soft fixed point that belongs to $A \cap B$.

Definition 2.2: Let $(\tilde{X}, \tilde{\rho}, E)$ be dislocated soft metric space and $U$ be a subset of $\tilde{X}$. We say that $U$ is $\tilde{\rho}$-open subset of $X$ if $\forall x \in \tilde{X}$, there exists $r>$ 0 such that $B_{\rho}(x, r) \subseteq U$. Also, $V \subseteq \tilde{X}$ is a $\tilde{\rho}$-closed subset of $\tilde{X}$ if $(\tilde{X} / V)$ is $\tilde{\rho}$-open subset of $\tilde{X}$.

Lemma 2.3: Let $(\tilde{X}, \tilde{\rho}, E)$ be dislocated soft metric space and $V$ be a $\tilde{\rho}$ closed subset of $X$. Let $\left\{x_{\lambda_{n}}^{n}\right\}$ be a sequence in $V$. If $x_{\lambda_{n}}^{n} \rightarrow x_{\lambda}$ as $n \rightarrow \infty$, then $x_{\lambda} \in V$.

Proof: Let $x_{\lambda} \notin V$, by definition 2.2, $X \backslash V$ is a $\tilde{\rho}$-open set then there exist $r>0$ such that $B_{\tilde{\rho}}\left(x_{\lambda}, r\right) \subseteq X \backslash V$. On the other hand, we have

$\lim _{n \rightarrow \infty}\left|\tilde{\rho}\left(x_{\lambda_{n}}^{n}, x_{\lambda}\right)-\tilde{\rho}\left(x_{\lambda}, x_{\lambda}\right)\right|=0$, Since $x_{\lambda_{n}}^{n} \rightarrow x_{\lambda}$ as $n \rightarrow \infty$. So there exist $n_{0} \in N$ such that

$$
\left|\tilde{\rho}\left(x_{\lambda_{n}}^{n}, x_{\lambda}\right)-\tilde{\rho}\left(x_{\lambda}, x_{\lambda}\right)\right|<r
$$

for all $n>n_{0}$. Hence we conclude that $\left\{x_{\lambda_{n}}^{n}\right\} \subseteq B_{\rho}\left(x_{\lambda}, r\right) \subseteq X \backslash V$, for all $n>n_{0}$. This is contradiction since $\left\{x_{\lambda_{n}}^{n}\right\} \subseteq V$, for all $n$ in $N$. 
Lemma 2.4: Let $(\tilde{X}, \tilde{\rho}, E)$ be dislocated soft metric space and $\left\{x_{\lambda_{n}}^{n}\right\}$ be a sequence in $(\tilde{X}, \tilde{\rho}, E)$ such that $\left\{x_{\lambda_{n}}^{n}\right\} \rightarrow x_{\lambda}$ as $n \rightarrow \infty$ and $\tilde{\rho}\left(x_{\lambda}, x_{\lambda}\right)=0$. Then

$$
\lim _{n \rightarrow \infty} \tilde{\rho}\left(x_{\lambda_{n}}^{n}, y_{\mu}\right)=\tilde{\rho}\left(x_{\lambda}, y_{\mu}\right) \text {, for all } y_{\mu} \in(\tilde{X}, \tilde{\rho}, E) \text {. }
$$

Lemma 2.5: If $(\tilde{X}, \tilde{\rho}, E)$ be soft dislocated metric space, then the following conditions holds

A. If $\tilde{\rho}\left(x_{\lambda_{n}}^{n}, x_{\lambda_{n}}^{n}\right)=0$, then $\tilde{\rho}\left(x_{\lambda} x_{\lambda}\right)=\tilde{\rho}\left(y_{\mu}, y_{\mu}\right)=0$;

B. If $\left\{x_{\lambda_{n}}^{n}\right\}$ be a sequence such that $\lim _{n \rightarrow \infty} \tilde{\rho}\left(x_{\lambda_{n}}^{n}, x_{\lambda_{n+1}}^{n+1}\right)=0$, then we have

$\lim _{n \rightarrow \infty} \tilde{\rho}\left(x_{\lambda_{n}}^{n}, x_{\lambda_{n}}^{n}\right)=\lim _{n \rightarrow \infty} \tilde{\rho}\left(x_{\lambda_{n+1}}^{n+1}, x_{\lambda_{n+1}}^{n+1}\right)=0 ;$

C. If $x_{\lambda} \neq y_{\mu}$, then $\tilde{\rho}\left(x_{\lambda}, y_{\mu}\right)>0$;

$\tilde{\rho}\left(x_{\lambda} x_{\lambda}\right)=\frac{2}{n} \sum_{i=1}^{n} \tilde{\rho}\left(x_{\lambda}, x_{\lambda_{i}}^{i}\right)$, holds for all $x_{\lambda}, x_{\lambda_{i}}^{i} \in \tilde{X}$, where $1 \leq i \leq n$.

D. At first we define the class of $\Phi$ and $\Psi$ by the following ways:

$\Psi=\{\psi:[0, \infty) \rightarrow[0, \infty)$ such that $\psi$ is non decreasing and continuous $\}$ and $\Phi=\{\phi:[0, \infty) \rightarrow[0, \infty)$ such that $\phi$ is lower semicontinuous $\}$.

Definition 2.6: Let $(\tilde{X}, \tilde{\rho}, E)$ be a soft dislocated metric space, $m \in N$, let $A_{1}, A_{2}, \cdots, A_{m}$ be $\tilde{\rho}$-closed non empty subsets of $\tilde{X}$ and let $\tilde{Y}=\bigcup_{i=1}^{i=m} A_{i}$. We say that $(f, \varphi)$ is a cyclic generalized $\phi-\psi$ contractive mapping if

i. $\quad \tilde{Y}=\bigcup_{i=1}^{i=m} A_{i}$ is a cyclic representation of $\tilde{Y}$ with respect to $(f, \varphi)$,

ii. $\psi(t)-\psi(s)+\phi(s)>0$ for all $t>0$ and $s=t$ or $s=0$ and

$$
\psi\left(\tilde{\rho}\left((f, \varphi) x_{\lambda},(f, \varphi) y_{\mu}\right)\right) \prec \psi\left(M_{\tilde{\rho}}\left(x_{\lambda}, y_{\mu}\right)\right)-\phi\left(M_{\tilde{\rho}}\left(x_{\lambda}, y_{\mu}\right)\right),
$$

for any $x_{\lambda} \in A_{i}, y_{\mu} \in A_{i+1}, \quad i=1,2,3, \cdots, m$, where $A_{i+1}=A_{1}, \phi \in \Phi, \psi \in \Psi$ and

$$
\begin{aligned}
M_{\tilde{\rho}}\left(x_{\lambda}, y_{\mu}\right)= & \max \left\{\tilde{\rho}\left(x_{\lambda}, y_{\mu}\right), \tilde{\rho}\left(x_{\lambda},(f, \varphi) x_{\lambda}\right), \tilde{\rho}\left(y_{\mu},(f, \varphi) y_{\mu}\right),\right. \\
& \left.\frac{\tilde{\rho}\left(y_{\mu},(f, \varphi) x_{\lambda}\right)+\tilde{\rho}\left(y_{\mu},(f, \varphi) y_{\mu}\right)+\tilde{\rho}\left(x_{\lambda},(f, \varphi) x_{\lambda}\right)}{6}\right\} .
\end{aligned}
$$

Let $\tilde{X}$ be a non-empty set and $(f, \varphi):(\tilde{X}, \tilde{\rho}, E) \rightarrow(\tilde{X}, \tilde{\rho}, E)$ be given map. The set of all soft fixed points of $(f, \varphi)$ will be denoted by $\operatorname{Fix}((f, \varphi))$ i.e. $\operatorname{Fix}((f, \varphi))=\left\{x_{\lambda} \in \tilde{X}: x_{\lambda}=(f, \varphi) x_{\lambda}\right\}$.

Theorem 2.7: Let $(\tilde{X}, \tilde{\rho}, E)$ be a complete dislocated soft metric space, $m \in N$, let $A_{1}, A_{2}, \cdots, A_{m}$ be non-empty $\tilde{\rho}$-closed subsets of $(\tilde{X}, \tilde{\rho}, E)$ and let $\tilde{Y}=\bigcup_{i=1}^{i=m} A_{i}$. Suppose that $(f, \varphi): \tilde{Y} \rightarrow \tilde{Y}$ is a cyclic generalized $\phi-\psi$ contractive mapping. Then $(f, \varphi)$ has fixed point in $\bigcap_{i=1}^{n} A_{i}$. Moreover if $\tilde{\rho}\left(x_{\lambda}, y_{\mu}\right) \geq \tilde{\rho}\left(x_{\lambda}, x_{\lambda}\right)$ for all $x, y \in \operatorname{Fix}(f, \varphi)$, then $(f, \varphi)$ has a unique fixed point in $\bigcap_{i=1}^{n} A_{i}$.

Proof: Let $x_{\lambda_{0}}^{0}$ be an arbitrary point of $\tilde{Y}$, so there exists some $i_{0}$ such that $x_{\lambda_{0}}^{0} \in A_{i_{0}}$. We know that $(f, \varphi)\left(A_{i_{0}}\right) \subseteq A_{i_{0}+1}$, we conclude that $(f, \varphi)\left(x_{\lambda_{0}}^{0}\right) \in A_{i_{0}+1}$. Thus there exist $x_{\lambda_{1}}^{1}$ in $A_{i_{0}+1}$ such that $(f, \varphi) x_{\lambda_{0}}^{0}=x_{\lambda_{1}}^{1}$. 
Recursive $(f, \varphi) x_{\lambda_{n}}^{n}=x_{\lambda_{n+1}}^{n+1}$, where $x_{\lambda_{n}}^{n} \in A_{i_{n}}$. Hence for $n \geq 0$ there exist $i_{n} \in\{1,2,3, \cdots, m\}$ such that $x_{\lambda_{n}}^{n} \in A_{i_{n}}$. In case $x_{\lambda_{n_{0}}}^{n_{0}}=x_{\lambda_{n_{0}+1}}^{n_{0}+1}$ for some $n_{0}=0,1,2, \cdots$, then it is clear that $x_{\lambda_{n_{0}}}^{n_{0}}$ is a soft fixed point of $(f, \varphi)$. Now assume that $x_{\lambda_{n}}^{n} \neq x_{\lambda_{n+1}^{n+1}}^{n}$ for all $n$. Hence by lemma 2.5(c) we have $\tilde{\rho}\left(x_{\lambda_{n-1}}^{n-1}, x_{\lambda_{n}}^{n}\right)>0$ for all $n$. We shall show that the sequence $\left\{d_{n}\right\}$ is non-increasing, where $d_{n}=\tilde{\rho}\left(x_{\lambda_{n}}^{n}, x_{\lambda_{n+1}}^{n+1}\right)$. Assume that there exists some $n_{0} \in N$ such that

$$
\tilde{\rho}\left(x_{\lambda_{n_{0}-1}^{n_{0}-1}}^{n_{1}}, x_{n_{0}}^{n_{0}}\right) \leq \tilde{\rho}\left(x_{\lambda_{n_{0}}}^{n_{0}}, x_{n_{0}+1}^{n_{0}+1}\right)
$$

Hence we get

$$
\psi\left(\tilde{\rho}\left(x_{n_{0}-1}^{n_{0}-1}, x_{n_{0}}^{n_{0}}\right)\right) \leq \psi\left(\tilde{\rho}\left(x_{n_{0}}^{n_{0}}, x_{n_{0}+1}^{n_{0}+1}\right)\right) .
$$

$$
\begin{aligned}
\text { Set } \tilde{x}_{\lambda_{1}}^{1}=(f, \varphi)\left(\tilde{x}_{\lambda}^{0}\right) & =\left(f\left(\tilde{x}_{\lambda}^{0}\right)\right)_{\varphi(\lambda)}, \tilde{x}_{\lambda_{2}}^{2}=(f, \varphi)\left(\tilde{x}_{\lambda_{1}}^{1}\right)=\left(f^{2}\left(\tilde{x}_{\lambda}^{0}\right)\right)_{\varphi^{2}(\lambda)}, \cdots, \\
\tilde{x}_{\lambda_{n+1}}^{n+1} & =(f, \varphi)\left(\tilde{x}_{\lambda_{n}}^{n}\right)=\left(f^{n+1}\left(\tilde{x}_{\lambda}^{0}\right)\right)_{\varphi^{n+1}(\lambda)}, \cdots
\end{aligned}
$$

Using conditions (1) together with (2), we get

$$
\begin{aligned}
& \psi\left(\tilde{\rho}\left(x_{\lambda_{n}}^{n}, x_{\lambda_{n+1}}^{n+1}\right)\right)=\psi\left(\tilde{\rho}\left((f, \varphi) x_{\lambda_{n-1}}^{n-1},(f, \varphi) x_{\lambda_{n}}^{n}\right)\right) \leq \tilde{\rho}\left((f, \varphi) x_{\lambda_{n-1}}^{n-1},(f, \varphi) x_{\lambda_{n}}^{n}\right) \\
& \leq \psi\left(\operatorname { m a x } \left\{\tilde{\rho}\left(x_{\lambda_{n-1}^{n-1}}^{n-1} x_{\lambda_{n}}^{n}\right), \tilde{\rho}\left(x_{\lambda_{n-1}^{n}}^{n-1},(f, \varphi) x_{\lambda_{n-1}^{n-1}}^{n-1}\right), \tilde{\rho}\left(x_{\lambda_{n}}^{n},(f, \varphi) x_{\lambda_{n}}^{n}\right),\right.\right. \\
& \left.\left.\frac{\tilde{\rho}\left(x_{\lambda_{n}}^{n},(f, \varphi) x_{\lambda_{n-1}^{n-1}}^{n-1}\right)+\tilde{\rho}\left(x_{\lambda_{n}}^{n},(f, \varphi) x_{\lambda_{n}}^{n}\right)+\tilde{\rho}\left(x_{\lambda_{n-1}^{n-1}}^{n-1},(f, \varphi) x_{\lambda_{n-1}^{n-1}}^{n-1}\right)}{6}\right\}\right) \\
& -\phi\left(\operatorname { m a x } \left\{\tilde{\rho}\left(x_{\lambda_{n-1}^{n-1}}^{n-1}, x_{\lambda_{n}}^{n}\right), \tilde{\rho}\left(x_{\lambda_{n-1}^{n-1}}^{n-1},(f, \varphi) x_{\lambda_{n-1}}^{n-1}\right), \tilde{\rho}\left(x_{\lambda_{n}}^{n},(f, \varphi) x_{\lambda_{n}}^{n}\right),\right.\right. \\
& \left.\left.\frac{\tilde{\rho}\left(x_{\lambda_{n}}^{n},(f, \varphi) x_{\lambda_{n-1}^{n-1}}^{n-1}\right)+\tilde{\rho}\left(x_{\lambda_{n}}^{n},(f, \varphi) x_{\lambda_{n}}^{n}\right)+\tilde{\rho}\left(x_{\lambda_{n-1}^{n-1}}^{n-1},(f, \varphi) x_{\lambda_{n-1}^{n-1}}^{n-1}\right)}{6}\right\}\right) \\
& \leq \psi\left(\max \left\{\tilde{\rho}\left(x_{\lambda_{n-1}}^{n-1}, x_{\lambda_{n}}^{n}\right), \tilde{\rho}\left(x_{\lambda_{n-1}}^{n-1}, x_{\lambda_{n}}^{n}\right), \tilde{\rho}\left(x_{\lambda_{n}}^{n}, x_{\lambda_{n+1}}^{n+1}\right), \frac{\tilde{\rho}\left(x_{\lambda_{n}}^{n}, x_{\lambda_{n}}^{n}\right)+\tilde{\rho}\left(x_{\lambda_{n}}^{n}, x_{\lambda_{n+1}}^{n+1}\right)+\tilde{\rho}\left(x_{\lambda_{n-1}}^{n-1}, x_{\lambda_{n}}^{n}\right)}{6}\right\}\right) \\
& -\phi\left(\max \left\{\tilde{\rho}\left(x_{\lambda_{n-1}^{n-1}}^{n-1} x_{\lambda_{n}}^{n}\right), \tilde{\rho}\left(x_{\lambda_{n-1}}^{n-1}, x_{\lambda_{n}}^{n}\right), \tilde{\rho}\left(x_{\lambda_{n}}^{n}, x_{\lambda_{n+1}}^{n+1}\right), \frac{\tilde{\rho}\left(x_{\lambda_{n}}^{n}, x_{\lambda_{n}}^{n}\right)+\tilde{\rho}\left(x_{\lambda_{n}}^{n}, x_{\lambda_{n+1}}^{n+1}\right)+\tilde{\rho}\left(x_{\lambda_{n-1}}^{n-1}, x_{\lambda_{n}}^{n}\right)}{6}\right\}\right) \\
& \leq \psi\left(\max \left\{\tilde{\rho}\left(x_{\lambda_{n-1}}^{n-1}, x_{\lambda_{n}}^{n}\right), \tilde{\rho}\left(x_{\lambda_{n}}^{n}, x_{\lambda_{n+1}}^{n+1}\right), \frac{\tilde{\rho}\left(x_{\lambda_{n}}^{n}, x_{\lambda_{n}}^{n}\right)+\tilde{\rho}\left(x_{\lambda_{n}}^{n}, x_{\lambda_{n+1}}^{n+1}\right)+\tilde{\rho}\left(x_{\lambda_{n-1}}^{n-1}, x_{\lambda_{n}}^{n}\right)}{6}\right\}\right) \\
& -\phi\left(\max \left\{\tilde{\rho}\left(x_{\lambda_{n-1}^{n-1}}^{n-1} x_{\lambda_{n}}^{n}\right), \tilde{\rho}\left(x_{\lambda_{n}}^{n}, x_{\lambda_{n+1}}^{n+1}\right), \frac{\tilde{\rho}\left(x_{\lambda_{n}}^{n}, x_{\lambda_{n}}^{n}\right)+\tilde{\rho}\left(x_{\lambda_{n}}^{n}, x_{\lambda_{n+1}}^{n+1}\right)+\tilde{\rho}\left(x_{\lambda_{n-1}^{n-1}}^{n-1} x_{\lambda_{n}}^{n}\right)}{6}\right\}\right) .
\end{aligned}
$$

On the other hand, from lemma 2.5(D) we have 


$$
\begin{aligned}
& \tilde{\rho}\left(x_{\lambda_{n}}^{n}, x_{\lambda_{n}}^{n}\right) \leq \tilde{\rho}\left(x_{\lambda_{n-1}}^{n-1}, x_{\lambda_{n}}^{n}\right)+\tilde{\rho}\left(x_{\lambda_{n}}^{n}, x_{\lambda_{n+1}}^{n+1}\right) \text { and } \\
& \frac{\tilde{\rho}\left(x_{\lambda_{n}}^{n}, x_{\lambda_{n}}^{n}\right)+\tilde{\rho}\left(x_{\lambda_{n}}^{n}, x_{\lambda_{n+1}^{n+1}}^{n+1}\right)+\tilde{\rho}\left(x_{\lambda_{n-1}}^{n-1}, x_{\lambda_{n}}^{n}\right)}{6} \leq \frac{\tilde{\rho}\left(x_{\lambda_{n-1}^{n-1}}^{n-} x_{\lambda_{n}}^{n}\right)+\tilde{\rho}\left(x_{\lambda_{n}}^{n}, x_{\lambda_{n+1}^{n+1}}^{n+1}\right)}{3} \text {. } \\
& \text { That is } \max \left\{\tilde{\rho}\left(x_{\lambda_{n-1}^{n-1}}^{n-1}, x_{\lambda_{n}}^{n}\right), \tilde{\rho}\left(x_{\lambda_{n}}^{n}, x_{\lambda_{n+1}}^{n+1}\right), \frac{\tilde{\rho}\left(x_{\lambda_{n-1}}^{n-1}, x_{\lambda_{n}}^{n}\right)+\tilde{\rho}\left(x_{\lambda_{n}}^{n}, x_{\lambda_{n+1}}^{n+1}\right)}{3}\right\} \\
& \leq \max \left\{\tilde{\rho}\left(x_{\lambda_{n-1}}^{n-1}, x_{\lambda_{n}}^{n}\right), \tilde{\rho}\left(x_{\lambda_{n}}^{n}, x_{\lambda_{n+1}}^{n+1}\right)\right\} .
\end{aligned}
$$

Therefore from (3) we get

$$
\begin{aligned}
\psi\left(\tilde{\rho}\left(x_{\lambda_{n}}^{n}, x_{\lambda_{n+1}}^{n+1}\right)\right) \leq & \psi\left(\max \left\{\tilde{\rho}\left(x_{\lambda_{n-1}^{n-1}}^{n-1}, x_{\lambda_{n}}^{n}\right), \tilde{\rho}\left(x_{\lambda_{n}}^{n}, x_{\lambda_{n+1}}^{n+1}\right)\right\}\right) \\
& -\phi\left(\max \left\{\tilde{\rho}\left(x_{\lambda_{n-1}^{n-1}}^{n-1}, x_{\lambda_{n}}^{n}\right), \tilde{\rho}\left(x_{\lambda_{n}}^{n}, x_{\lambda_{n+1}}^{n+1}\right)\right\}\right) .
\end{aligned}
$$

Now, if $\max \left\{\tilde{\rho}\left(x_{\lambda_{n-1}}^{n-1}, x_{\lambda_{n}}^{n}\right), \tilde{\rho}\left(x_{\lambda_{n}}^{n}, x_{\lambda_{n+1}}^{n+1}\right)\right\}=\tilde{\rho}\left(x_{\lambda_{n}}^{n}, x_{\lambda_{n+1}}^{n+1}\right)$, then

$$
\tilde{\rho}\left(x_{\lambda_{n}}^{n}, x_{\lambda_{n+1}}^{n+1}\right) \leq \alpha \tilde{\rho}\left(x_{\lambda_{n}}^{n}, x_{\lambda_{n+1}}^{n+1}\right)-\beta \tilde{\rho}\left(x_{\lambda_{n}}^{n}, x_{\lambda_{n+1}}^{n+1}\right) .
$$

This is contradiction. Hence we have

$$
\psi \tilde{\rho}\left(x_{\lambda_{n}}^{n}, x_{\lambda_{n+1}}^{n+1}\right) \leq \psi\left\{\tilde{\rho}\left(x_{\lambda_{n-1}^{n-1}}^{n-1} x_{\lambda_{n}}^{n}\right)\right\}-\phi\left\{\tilde{\rho}\left(x_{\lambda_{n-1}}^{n-1}, x_{\lambda_{n}}^{n}\right)\right\},
$$

for all $n \in N$. By taking $x_{\lambda}=x_{\lambda_{n_{0}-1}}^{n_{0}-1}$ and $y_{\mu}=x_{\lambda_{n_{0}}}^{n_{0}}$ in (4) and keeping (2) in mind, we deduce that

$$
\psi \tilde{\rho}\left(x_{\lambda_{n_{0}-1}}^{n_{0}-1}, x_{\lambda_{n_{0}}}^{n_{0}}\right) \leq \psi\left\{\tilde{\rho}\left(x_{\lambda_{n_{0}-1}^{n_{0}-1}}, x_{\lambda_{n_{0}}}^{n_{0}}\right)\right\}-\phi\left\{\tilde{\rho}\left(x_{\lambda_{n_{0}-1}}^{n_{0}-1}, x_{\lambda_{n_{0}}}^{n_{0}}\right)\right\} .
$$

This is a contradiction. Hence we conclude that $d_{n}<d_{n-1}$ i.e. $\tilde{\rho}\left(x_{\lambda_{n}}^{n}, x_{\lambda_{n+1}}^{n+1}\right) \leq \tilde{\rho}\left(x_{\lambda_{n-1}^{n-1}}^{n-1}, x_{\lambda_{n}}^{n}\right)$ hold for all $n \in N$. Thus there exist $r \geq 0$ such that $\lim _{n \rightarrow \infty} d_{n}=\lim _{n \rightarrow \infty} \tilde{\rho}\left(x_{\lambda_{n}}^{n}, x_{\lambda_{n+1}}^{n+1}\right)=r$. We shall show that $r=0$ by the method of reductio ad absurdum. For this purpose, we assume that $r>0$. By (4) together with the property of $\phi$ and $\psi$ we have

$$
\psi(r)=\lim _{n \rightarrow \infty} \sup \psi\left(d_{n}\right) \leq \lim _{n \rightarrow \infty} \sup \left[\psi\left(d_{n-1}\right)-\phi\left(d_{n-1}\right)\right] \leq \psi(r)-\phi(r) .
$$

This yields that $\varphi(r) \leq 0$. This is contradiction. Hence we obtain that

$$
\lim _{n \rightarrow \infty} d_{n} \leq \lim _{n \rightarrow \infty}\left(x_{\lambda_{n}}^{n}, x_{\lambda_{n+1}^{n+1}}^{n+1}\right)=0 .
$$

We shall show that $\left\{x_{\lambda_{n}}^{n}\right\}$ is a $\tilde{\rho}$-Cauchy sequence. To reach this goal, first we prove the followings claim:

(k) For every $\epsilon>0$, there exists $n \in N$ such that if $r, q \geq n$ with $r-q \equiv 1(m)$, then $\tilde{\rho}\left(x_{\lambda_{r_{n}}}^{r_{n}}, x_{\lambda_{q_{n}}}^{q_{n}}\right)<\epsilon$.

Suppose, on the contrary that there exist $\epsilon>0$ such that for any $n \in N$, we can find $r_{n}>q_{n} \geq n$ with $r_{n}-q_{n} \equiv 1(m)$ satisfying

$$
\tilde{\rho}\left(x_{\lambda_{q_{n}}}^{q_{n}}, x_{\lambda_{r_{n}}}^{r_{n}}\right) \geq \epsilon .
$$

Now we consider $n>2 m$. Then, corresponding to $q_{n} \geq n$, we can choose $r_{n}$ in such a way that it is the smallest integer with $r_{n}>q_{n}$ satisfying 
$r_{n}-q_{n} \equiv 1(m)$ and $\tilde{\rho}\left(x_{\lambda_{q_{n}}}^{q_{n}}, x_{\lambda_{r_{n}}}^{r_{n}}\right) \geq \epsilon$. Therefore $\tilde{\rho}\left(x_{\lambda_{q_{n}}}^{q_{n}}, x_{\lambda_{n_{n}}-m}^{r_{n}-m}\right) \leq \epsilon$. By using triangular inequality, we obtain

$$
\begin{aligned}
\epsilon & \leq \tilde{\rho}\left(x_{\lambda_{q_{n}}}^{q_{n}}, x_{\lambda_{r_{n}}}^{r_{n}}\right) \\
& \leq \tilde{\rho}\left(x_{\lambda_{q_{n}}}^{q_{n}}, x_{\lambda_{q_{n}-m}}^{q_{n}-m}\right)+\sum_{i=1}^{m} \tilde{\rho}\left(x_{\lambda_{r_{n}-i}}^{r_{n}-i}, x_{\lambda_{r_{n}-i+1}}^{r_{n}-i+1}\right) . \\
& \leq \epsilon+\sum_{i=1}^{m} \tilde{\rho}\left(x_{\lambda_{r_{n}-i}}^{r_{n}-i}, x_{\lambda_{r_{n}-i+1}}^{r_{n}-i+1}\right)
\end{aligned}
$$

Passing to the limit as $n \rightarrow \infty$ in the last inequality and taking (5) into account, we obtain that

$$
\lim _{n \rightarrow \infty} \tilde{\rho}\left(x_{\lambda_{q_{n}}}^{q_{q_{n}}}, x_{\lambda_{r_{n}}}^{r_{n}}\right)=\epsilon
$$

Again by $\left(d_{3}\right)$ we derive that

$$
\begin{aligned}
& \epsilon \leq \tilde{\rho}\left(x_{\lambda_{q_{n}}}^{q_{n}}, x_{\lambda_{r_{n}}}^{r_{n}}\right) \\
& \leq \tilde{\rho}\left(x_{\lambda_{q_{n}}}^{q_{n}}, x_{\lambda_{q_{n+1}}}^{q_{n+1}}\right)+\tilde{\rho}\left(x_{\lambda_{q_{n+1}}}^{q_{n+1}}, x_{\lambda_{r_{n}+1}}^{r_{n}+1}\right)+\tilde{\rho}\left(x_{\lambda_{r_{n}+1}}^{r_{n}+1}, x_{\lambda_{r_{n}}}^{r_{n}}\right) \\
& \leq \tilde{\rho}\left(x_{\lambda_{q_{n}}}^{q_{n}}, x_{\lambda_{q_{n+1}}}^{q_{n+1}}\right)+\tilde{\rho}\left(x_{\lambda_{q_{n+1}}}^{q_{n+1}}, x_{\lambda_{q_{n}}}^{q_{n}}\right)+\tilde{\rho}\left(x_{\lambda_{q_{n}}}^{q_{q_{n}}}, x_{\lambda_{r_{n}}}^{r_{n}}\right) \\
& +\tilde{\rho}\left(x_{\lambda_{r_{n}}}^{r_{n}}, x_{\lambda_{r_{n}}+1}^{r_{n}+1}\right)+\tilde{\rho}\left(x_{\lambda_{r_{n}}+1}^{r_{n}+1}, x_{\lambda_{r_{n}}}^{r_{n}}\right) \\
& \leq 2 \tilde{\rho}\left(x_{\lambda_{q_{n}}}^{q_{n}}, x_{\lambda_{q_{n+1}}}^{q_{n+1}}\right)+\tilde{\rho}\left(x_{\lambda_{q_{n}}}^{q_{n}}, x_{\lambda_{r_{n}}}^{r_{n}}\right)+2 \tilde{\rho}\left(x_{\lambda_{r_{n}}+1}^{r_{n}+1}, x_{\lambda_{r_{n}}}^{r_{n}}\right) .
\end{aligned}
$$

Taking (5) and (7) in account we get

$$
\lim _{n \rightarrow \infty} \tilde{\rho}\left(x_{\lambda_{q_{n+1}}}^{q_{n+1}}, x_{\lambda_{r_{n}+1}}^{r_{n}+1}\right)=\epsilon
$$

By $\left(d_{3}\right)$ we have the following inequality

$$
\begin{array}{r}
\tilde{\rho}\left(x_{\lambda_{q_{n}}}^{q_{n}}, x_{\lambda_{r_{n}+1}}^{r_{n}+1}\right) \leq \tilde{\rho}\left(x_{\lambda_{q_{n}}}^{q_{n}}, x_{\lambda_{r_{n}}}^{r_{n}}\right)+\tilde{\rho}\left(x_{\lambda_{r_{n}}}^{r_{n}}, x_{\lambda_{r_{n}+1}}^{r_{n}+1}\right) \\
\text { and } \tilde{\rho}\left(x_{\lambda_{q_{n}}}^{q_{n}}, x_{\lambda_{r_{n}}}^{r_{n}}\right) \leq \tilde{\rho}\left(x_{\lambda_{q_{n}}}^{q_{q_{n}}}, x_{\lambda_{r_{n}+1}}^{r_{n}+1}\right)+\tilde{\rho}\left(x_{\lambda_{r_{n}}}^{r_{n}}, x_{\lambda_{r_{n}+1}}^{r_{n}+1}\right) .
\end{array}
$$

Letting $\lim n \rightarrow \infty$ in (9) and (10), we have

$$
\lim _{n \rightarrow \infty} \tilde{\rho}\left(x_{\lambda_{q_{n}}}^{q_{n}}, x_{\lambda_{r_{n}+1}}^{r_{n}+1}\right)=\epsilon
$$

Again by $\left(d_{3}\right)$ we have

$$
\begin{gathered}
\tilde{\rho}\left(x_{\lambda_{r_{n}}}^{r_{n}}, x_{\lambda_{q_{n}+1}}^{q_{n}+1}\right) \leq \tilde{\rho}\left(x_{\lambda_{r_{n}}}^{r_{n}}, x_{\lambda_{r_{n}+1}}^{r_{n}+1}\right)+\tilde{\rho}\left(x_{\lambda_{r_{n}+1}}^{r_{n}+1}, x_{\lambda_{q_{n}+1}}^{q_{n}+1}\right) \\
\text { and } \tilde{\rho}\left(x_{\lambda_{n_{n}+1}}^{r_{n}+1}, x_{\lambda_{q_{n}+1}}^{q_{n}+1}\right) \leq \tilde{\rho}\left(x_{\lambda_{r_{n}+1}}^{r_{n}+1}, x_{\lambda_{r_{n}}}^{r_{n}}\right)+\tilde{\rho}\left(x_{\lambda_{r_{n}}}^{r_{n}}, x_{\lambda_{q_{n}+1}}^{q_{n}+1}\right) .
\end{gathered}
$$

Letting $n \rightarrow \infty$ in (12) and (13), we derive that

$$
\lim _{n \rightarrow \infty} \tilde{\rho}\left(x_{\lambda_{r_{n}}}^{r_{n}}, x_{\lambda_{q_{n}+1}}^{q_{n}+1}\right)=\epsilon .
$$

Since $x_{\lambda_{q_{n}}}^{q_{n}}$ and $x_{\lambda_{r_{n}}}^{r_{n_{n}}}$ lie in different adjacently labeled sets $A_{\mathrm{i}}$ and $\mathrm{A}_{\mathrm{i}+1}$, for certain $1 \leq i \leq m$. By using (5), (7), (8), (11) and (14) together with the fact that $(f, \varphi)$ is a generalized cyclic $\phi-\psi$ contractive mappings, we find that 


$$
\begin{aligned}
& \psi\left(\tilde{\rho}\left(x_{\lambda_{q_{n}+1}}^{q_{n}+1}, x_{\lambda_{r_{n}+1}}^{r_{n}+1},\right)\right)=\psi\left(\tilde{\rho}\left((f, \varphi) x_{\lambda_{q_{n}}}^{q_{n}},(f, \varphi) x_{\lambda_{r_{n}}}^{r_{n}}\right)\right) \\
& \leq \psi\left(\operatorname { m a x } \left\{\tilde{\rho}\left(x_{\lambda_{q_{n}}}^{q_{n}}, x_{\lambda_{r_{n}}}^{r_{n}}\right), \tilde{\rho}\left(x_{\lambda_{q_{n}}}^{q_{n}},(f, \varphi) x_{\lambda_{q_{n}}}^{q_{n}}\right), \tilde{\rho}\left(x_{\lambda_{r_{n}}}^{r_{n}},(f, \varphi) x_{\lambda_{r_{n}}}^{r_{n}}\right),\right.\right. \\
& \left.\left.\frac{\tilde{\rho}\left(x_{\lambda_{r_{n}}}^{r_{n}},(f, \varphi) x_{\lambda_{q_{n}}}^{q_{n}}\right)+\tilde{\rho}\left(x_{\lambda_{r_{n}}}^{r_{n}},(f, \varphi) x_{\lambda_{r_{n}}}^{r_{n}}\right)+\tilde{\rho}\left(x_{\lambda_{q_{n}}}^{q_{n}},(f, \varphi) x_{\lambda_{q_{n}}}^{q_{n}}\right)}{6}\right\}\right) \\
& -\phi\left(\operatorname { m a x } \left\{\tilde{\rho}\left(x_{\lambda_{q_{n}}}^{q_{n}}, x_{\lambda_{r_{n}}}^{r_{n}}\right), \tilde{\rho}\left(x_{\lambda_{q_{n}}}^{q_{n}},(f, \varphi) x_{\lambda_{q_{n}}}^{q_{n}}\right), \tilde{\rho}\left(x_{\lambda_{r_{n}}}^{r_{n}},(f, \varphi) x_{\lambda_{r_{n}}}^{r_{n}}\right),\right.\right. \\
& \left.\left.\frac{\tilde{\rho}\left(x_{\lambda_{r_{n}}}^{r_{n}},(f, \varphi) x_{\lambda_{q_{n}}}^{q_{n}}\right)+\tilde{\rho}\left(x_{\lambda_{r_{n}}}^{r_{n}},(f, \varphi) x_{\lambda_{r_{n}}}^{r_{n}}\right)+\tilde{\rho}\left(x_{\lambda_{q_{n}}}^{q_{n}},(f, \varphi) x_{\lambda_{q_{n}}}^{q_{n}}\right)}{6}\right\}\right) \\
& \leq \psi\left(\operatorname { m a x } \left\{\tilde{\rho}\left(x_{\lambda_{q_{n}}^{q_{n}},}^{q_{\lambda_{r_{n}}}}\right), \tilde{\rho}\left(x_{\lambda_{q_{n}}}^{q_{n}}, x_{\lambda_{q_{n+1}}}^{q_{n_{n+1}}}\right), \tilde{\rho}\left(x_{\lambda_{r_{n}}}^{r_{n}}, x_{\lambda_{r_{n}+1}}^{r_{n+1}}\right),\right.\right. \\
& \left.\left.\frac{\tilde{\rho}\left(x_{\lambda_{r_{n}}}^{r_{n}}, x_{\lambda_{q_{n+1}}}^{q_{n+1}}\right)+\tilde{\rho}\left(x_{\lambda_{r_{n}}}^{r_{n}}, x_{\lambda_{r_{n}+1}}^{r_{n+1}}\right)+\tilde{\rho}\left(x_{\lambda_{q_{n}}}^{q_{q_{n}}}, x_{\lambda_{q_{n+1}}}^{q_{n+1}}\right)}{6}\right\}\right) \\
& -\phi\left(\operatorname { m a x } \left\{\tilde{\rho}\left(x_{\lambda_{q_{n}}}^{q_{n}}, x_{\lambda_{r_{n}}}^{r_{n}}\right), \tilde{\rho}\left(x_{\lambda_{q_{n}}}^{q_{n}}, x_{\lambda_{q_{n+1}}}^{q_{n+1}}\right), \tilde{\rho}\left(x_{\lambda_{r_{n}}}^{r_{n}}, x_{\lambda_{r_{n}+1}}^{r_{n+1}}\right),\right.\right. \\
& \left.\frac{\tilde{\rho}\left(x_{\lambda_{r_{n}}}^{r_{n}}, x_{\lambda_{n_{n+1}}}^{q_{q_{n+1}}}\right)+\tilde{\rho}\left(x_{\lambda_{r_{n}}}^{r_{n}}, x_{\lambda_{r_{n}+1}}^{r_{n+1}}\right)+\tilde{\rho}\left(x_{\lambda_{q_{n}}}^{q_{n}}, x_{\lambda_{q_{n+1}}}^{q_{n+1}}\right)}{6}\right\} .
\end{aligned}
$$

Regarding the property of $\phi$ and $\psi$ in the last inequality, we obtain that $\psi(\epsilon) \leq \psi(\epsilon)-\phi(\epsilon)$, which is a contradiction. Hence the condition (k) is a satisfied. Fix $\epsilon>0$. By the claim we find $n_{0} \in N$ such that if $r, q \geq n_{0}$ with $r-q \equiv 1(m)$,

$$
\tilde{\rho}\left(x_{\lambda_{r}}^{r}, x_{\lambda_{q}}^{q}\right) \leq \frac{\epsilon}{2} .
$$

Since $\lim _{n \rightarrow \infty} \tilde{d}\left(x_{\lambda_{n}}^{n}, x_{\lambda_{n+1}}^{n+1}\right)=0$, we also find $n_{1} \in N$ such that

$$
\tilde{\rho}\left(x_{\lambda_{n}}^{n}, x_{\lambda_{n+1}}^{n+1}\right) \leq \frac{\epsilon}{2 m},
$$

for any $n \geq n_{1}$. Suppose that $r, s \geq \max \left\{n_{0}, n_{1}\right\}$ and $s>r$. There exist $k \in\{1,2,3, \cdots, m\}$ such that $s-r \equiv k(m)$. Therefore $s-r+\phi \equiv 1(m)$, for $\phi=m-k+1$.

So we have for $j \in\{1,2,3, \cdots, m\}$ and $s+j-r \equiv 1(m)$,

$$
\tilde{\rho}\left(x_{\lambda_{r}}^{r}, x_{\lambda_{s}}^{s}\right) \leq \tilde{\rho}\left(x_{\lambda_{r}}^{r}, x_{\lambda_{s+j}}^{s+j}\right)+\tilde{\rho}\left(x_{\lambda_{s+j}}^{s+j}, x_{\lambda_{s+j-1}}^{s+j-1}\right)+\cdots+\tilde{\rho}\left(x_{\lambda_{s+1}}^{s+1}, x_{\lambda_{s}}^{s}\right) .
$$

By (15) \& (16) and from the last inequality, we get

$$
\tilde{\rho}\left(x_{\lambda_{r}}^{r}, x_{\lambda_{s}}^{s}\right) \leq \frac{\epsilon}{2}+j \times \frac{\epsilon}{2 m} \leq \frac{\epsilon}{2}+m \times \frac{\epsilon}{2 m}=\epsilon .
$$

This proves that $\left\{x_{\lambda_{n}}^{n}\right\}$ is a $\tilde{\rho}$-Cauchy sequence. Since $\epsilon$ is arbitrary, 
$\left\{x_{\lambda_{n}}^{n}\right\}$ is a Cauchy sequence. Since $Y$ is $\tilde{\rho}$-closed in $(\tilde{X}, \tilde{\rho}, E)$, then $(\tilde{Y}, \tilde{\rho}, E)$ is also complete, there exists $x_{\lambda} \in \tilde{Y}=\bigcup_{i=1}^{m} A_{i}$ such that $\lim _{n \rightarrow \infty} x_{\lambda_{n}}^{n}=x_{\lambda}$ in $(\tilde{Y}, \tilde{\rho}, E)$; equivalently

$$
\tilde{\rho}\left(x_{\lambda}, x_{\lambda}\right)=\lim _{n \rightarrow \infty} \tilde{\rho}\left(x_{\lambda}, x_{\lambda_{n}}^{n}\right)=\lim _{m, n \rightarrow \infty} \tilde{\rho}\left(x_{\lambda_{n}}^{n}, x_{\lambda_{m}}^{m}\right)=0 .
$$

In what follows, we prove that $x_{\lambda}$ is a soft fixed point of $(f, \varphi)$. In fact, since $\lim _{n \rightarrow \infty} x_{\lambda_{n}}^{n}=x_{\lambda}$ and $\tilde{Y}=\bigcup_{i=1}^{m} A_{i}$ is a cyclic representation of $Y$ with respect to $(f, \varphi)$. The sequence $\left\{x_{\lambda_{n}}^{n}\right\}$ has infinite terms in each $A_{i}$, for $i \in\{1,2,3, \cdots, m\}$. Suppose that $x_{\lambda} \in A_{i}, \quad(f, \varphi) x_{\lambda} \in A_{i+1}$ and we take a subsequence $\left\{x_{\lambda_{n_{k}}}^{n_{k}}\right\}$ of $\left\{x_{\lambda_{n}}^{n}\right\}$ with $x_{\lambda_{n_{k}}}^{n_{k}} \in A_{i-1}$ (the existence of this subsequence is guaranteed by abovementioned comment). By using the contractive condition we can obtain

$$
\begin{aligned}
& \psi\left(\tilde{\rho}\left((f, \varphi) x_{\lambda},(f, \varphi) x_{\lambda_{n k}}^{n_{k}}\right)\right) \\
& \leq \psi\left(\operatorname { m a x } \left\{\tilde{\rho}\left(x_{\lambda}, x_{\lambda_{n k}}^{n_{k}}\right), \tilde{\rho}\left(x_{\lambda},(f, \varphi) x_{\lambda}\right), \tilde{\rho}\left(x_{\lambda_{n k}}^{n_{k}},(f, \varphi) x_{\lambda_{n k}}^{n_{k}}\right),\right.\right. \\
& \left.\left.\frac{\left.\left.\tilde{\rho}\left(x_{\lambda_{n k}}^{n_{k}},(f, \varphi) x_{\lambda}\right)+\tilde{\rho}\left(x_{\lambda_{n k}}^{n_{k}},(f, \varphi) x_{\lambda_{n k}}^{n_{k}}\right)+\tilde{\rho}\left(x_{\lambda},(f, \varphi) x_{\lambda}\right)\right\}\right)}{6}\right\}\right), \\
& \quad-\phi\left(\operatorname { m a x } \left\{\tilde{\rho}\left(x_{\lambda}, x_{\lambda_{n k}}^{n_{k}}\right), \tilde{\rho}\left(x_{\lambda},(f, \varphi) x_{\lambda}\right), \tilde{\rho}\left(x_{\lambda_{n k}}^{n_{k}},(f, \varphi) x_{\lambda_{n k}}^{n_{k}}\right),\right.\right. \\
& \left.\left.\frac{\tilde{\rho}\left(x_{\lambda_{n k}}^{n_{k}},(f, \varphi) x_{\lambda}\right)+\tilde{\rho}\left(x_{\lambda_{n k}}^{n_{k}},(f, \varphi) x_{\lambda_{n k}}^{n_{k}}\right)+\tilde{\rho}\left(x_{\lambda},(f, \varphi) x_{\lambda}\right)}{6}\right\}\right) .
\end{aligned}
$$

Passing to the limit as $n \rightarrow \infty$ and using $x_{\lambda_{n k}}^{n_{k}} \rightarrow x_{\lambda}$, lower semi-continuity of $\phi$, we have

$$
\psi\left(\tilde{\rho}\left(x_{\lambda},(f, \varphi) x_{\lambda}\right)\right) \leq \psi\left(\tilde{\rho}\left(x_{\lambda},(f, \varphi) x_{\lambda}\right)\right)-\phi\left(\tilde{\rho}\left(x_{\lambda},(f, \varphi) x_{\lambda}\right)\right) .
$$

So, $\tilde{\rho}\left(x_{\lambda},(f, \varphi) x_{\lambda}\right)=0$. Therefore $x_{\lambda}$ is a soft fixed point of $(f, \varphi)$. Finally to prove the uniqueness of soft fixed point, suppose that $y_{\mu}, z_{w} \in(\tilde{X}, \tilde{\rho}, E)$ are two distinct soft fixed points of $(f, \varphi)$. The cyclic character of $(f, \varphi)$ and the fact that $y_{\mu}, z_{w} \in(\tilde{X}, \tilde{\rho}, E)$ are soft fixed points of $(f, \varphi)$ implies that $x_{\lambda}, y_{\mu} \in \bigcap_{i=1}^{m} A_{i}$. Suppose that $x_{\lambda} \neq y_{\mu}$ and for all $p_{\lambda}, q_{\theta} \in \operatorname{Fix}((f, \varphi))$, $\tilde{\rho}\left(p_{\lambda}, q_{\theta}\right) \geq \tilde{\rho}\left(p_{\lambda}, p_{\lambda}\right)$. Using the contractive condition, we obtain

$$
\begin{aligned}
& \psi\left(\tilde{\rho}\left((f, \varphi) x_{\lambda},(f, \varphi) y_{\mu}\right)\right) \\
& \leq \psi\left(\operatorname { m a x } \left\{\tilde{\rho}\left(x_{\lambda}, y_{\mu}\right), \tilde{\rho}\left(x_{\lambda},(f, \varphi) x_{\lambda}\right), \tilde{\rho}\left(y_{\mu},(f, \varphi) y_{\mu}\right),\right.\right. \\
& \left.\left.\frac{\left.\left.\tilde{\rho}\left(y_{\mu},(f, \varphi) x_{\lambda}\right)+\tilde{\rho}\left(y_{\mu},(f, \varphi) y_{\mu}\right)+\tilde{\rho}\left(x_{\lambda},(f, \varphi) x_{\lambda}\right)\right\}\right)}{6}\right\}\right) \\
& -\phi\left(\operatorname { m a x } \left\{\tilde{\rho}\left(x_{\lambda}, y_{\mu}\right), \tilde{\rho}\left(x_{\lambda},(f, \varphi) x_{\lambda}\right), \tilde{\rho}\left(y_{\mu},(f, \varphi) y_{\mu}\right),\right.\right. \\
& \left.\left.\frac{\left.\left.\tilde{\rho}\left(y_{\mu},(f, \varphi) x_{\lambda}\right)+\tilde{\rho}\left(y_{\mu},(f, \varphi) y_{\mu}\right)+\tilde{\rho}\left(x_{\lambda},(f, \varphi) x_{\lambda}\right)\right\}\right)}{6}\right\}\right)
\end{aligned}
$$


Then we have $\psi\left(\tilde{\rho}\left(x_{\lambda}, y_{\mu}\right)\right) \leq \psi\left(\tilde{\rho}\left(x_{\lambda}, y_{\mu}\right)\right)-\phi\left(\tilde{\rho}\left(x_{\lambda}, y_{\mu}\right)\right)$.

This is a contradiction. Thus we derive that $\tilde{\rho}\left(y_{\mu}, z_{w}\right)=0 \Leftrightarrow y_{\mu}=z_{w}$. Hence proved.

In the theorem 2.7, if we take $(\tilde{X}, \tilde{\rho}, E)=A_{i}$, for all $0 \leq i \leq m$, then we deduce the following theorem.

Theorem 2.8: Let $(\tilde{X}, \tilde{\rho}, E)$ be a complete soft dislocated metric space and $(f, \varphi)$ be self map on $(\tilde{X}, \tilde{\rho}, E)$. Assume that there exist $\varphi \in \Phi, \psi \in \Psi$ such that

$\psi\left(\tilde{\rho}\left((f, \varphi) x_{\lambda},(f, \varphi) y_{\mu}\right)\right) \leq \psi\left(M_{\tilde{\rho}}\left(x_{\lambda}, y_{\mu}\right)\right)-\phi\left(M_{\tilde{\rho}}\left(x_{\lambda}, y_{\mu}\right)\right)$, for all $x_{\lambda}, y_{\mu} \in(\tilde{X}, \tilde{\rho}, E)$, where

$$
\begin{aligned}
M_{\tilde{\rho}}\left(x_{\lambda}, y_{\mu}\right)= & \max \left\{\tilde{\rho}\left(x_{\lambda}, y_{\mu}\right), \tilde{\rho}\left(x_{\lambda},(f, \varphi) x_{\lambda}\right), \tilde{\rho}\left(y_{\mu},(f, \varphi) y_{\mu}\right),\right. \\
& \left.\frac{\tilde{\rho}\left(y_{\mu},(f, \varphi) x_{\lambda}\right)+\tilde{\rho}\left(y_{\mu},(f, \varphi) y_{\mu}\right)+\tilde{\rho}\left(x_{\lambda},(f, \varphi) x_{\lambda}\right)}{6}\right\} .
\end{aligned}
$$

Then $(f, \varphi)$ has a soft fixed point. Moreover if $\tilde{\rho}\left(x_{\lambda}, y_{\mu}\right) \geq \tilde{\rho}\left(x_{\lambda}, x_{\lambda}\right)$ for all $x_{\lambda}, y_{\mu} \in \operatorname{Fix}((f, \varphi))$, then $(f, \varphi)$ has a unique soft fixed point.

If in theorem (2.7) we take $\psi(t)=t$ and $\phi(t)=(1-r) t$, where $r \in[0,1)$ then we deduce the following corollary.

Corollary 2.9: Let $(\tilde{X}, \tilde{\rho}, E)$ be a complete soft dislocated metric space, $m \in N$, let $A_{1}, A_{2}, \cdots, A_{m}$ be non empty $\tilde{\rho}$-closed subsets of $(\tilde{X}, \tilde{\rho}, E)$ and let $Y=\bigcup_{i=1}^{i=m} A_{i}$. Suppose that $(f, \varphi): Y \rightarrow Y$ is an operator such that

i. $Y=\bigcup_{i=1}^{i=m} A_{i}$ is a cyclic representation of $(\tilde{X}, \tilde{\rho}, E)$ with respect to $(f, \varphi)$, ii. there exist $r \in[0,1)$ such that

$$
\begin{aligned}
\tilde{\rho}\left((f, \varphi) x_{\lambda},(f, \varphi) y_{\mu}\right) & \\
= & r \max \left\{\tilde{\rho}\left(x_{\lambda}, y_{\mu}\right), \tilde{\rho}\left(x_{\lambda},(f, \varphi) x_{\lambda}\right), \tilde{\rho}\left(y_{\mu},(f, \varphi) y_{\mu}\right),\right. \\
& \left.\frac{\tilde{\rho}\left(y_{\mu},(f, \varphi) x_{\lambda}\right)+\tilde{\rho}\left(y_{\mu},(f, \varphi) y_{\mu}\right)+\tilde{\rho}\left(x_{\lambda},(f, \varphi) x_{\lambda}\right)}{6}\right\},
\end{aligned}
$$

for any $x \in A_{i}$ and $y \in A_{i+1}, \quad i=1,2,3, \cdots, m$. Where $A_{m+1}=A_{1}$, then $(f, \varphi)$ has a soft fixed point $z_{w} \in \bigcap_{i=1}^{m} A_{i}$. Moreover if $\tilde{\rho}\left(x_{\lambda}, y_{\mu}\right) \geq \tilde{\rho}\left(x_{\lambda}, x_{\lambda}\right)$ for all $x_{\lambda} \in \operatorname{Fix}(f, \varphi)$, then $(f, \varphi)$ has a unique soft fixed point.

Example 2.10: Let $X=R$ with soft dislocated metric $\tilde{\rho}\left(x_{\lambda}, y_{\mu}\right)=\max \left\{\left|\frac{x_{\lambda}}{2}\right|,\left|\frac{y_{\mu}}{2}\right|\right\}$, for all $x_{\lambda}, y_{\mu} \in \bar{X}$. Suppose $A_{1}=[-2,0]$, $A_{2}=[0,2] \& Y=\bigcup_{i=1}^{i=2} A_{i}$. Define $(f, \varphi): Y \rightarrow Y$ by

$$
(f, \varphi)= \begin{cases}\frac{\left(x_{\lambda}\right)^{2}}{8}, & \text { if } x_{\lambda} \in[-2,0] \\ \frac{-x_{\lambda}}{5}, & \text { if } x_{\lambda} \in[0,2]\end{cases}
$$

It is clear that $\bigcup_{i=1}^{i=2} A_{i}$ is a cyclic representation of $Y$ with respect to $(f, \varphi)$. 
Let $x_{\lambda} \in A_{1}=[-2,0]$ and $y_{\mu} \in A_{2}=[0,2]$ then

$$
\begin{aligned}
& \tilde{\rho}\left((f, \varphi) x_{\lambda},(f, \varphi) y_{\mu}\right) \\
& =\tilde{\rho}\left(\frac{x_{\lambda}^{2}}{8}, \frac{-y_{\mu}}{5}\right)=\max \left\{\left|\frac{x_{\lambda}^{2} / 8}{2}\right|,\left|\frac{-y_{\mu} / 5}{2}\right|\right\}=\max \left\{\left|\frac{x_{\lambda}^{2}}{16}\right|,\left|\frac{-y_{\mu}}{10}\right|\right\} \\
& \leq \max \left\{\frac{x_{\lambda}}{4}, \frac{y_{\mu}}{4}\right\} \leq \frac{1}{2} \max \left\{\frac{x_{\lambda}}{2}, \frac{y_{\mu}}{2}\right\} \leq \frac{1}{2} \tilde{\rho}\left(x_{\lambda}, y_{\mu}\right) .
\end{aligned}
$$

and so

$$
\begin{aligned}
\tilde{\rho}\left((f, \varphi) x_{\lambda},(f, \varphi) y_{\mu}\right) & \\
= & r \max \left\{\tilde{\rho}\left(x_{\lambda}, y_{\mu}\right), \tilde{\rho}\left(x_{\lambda},(f, \varphi) x_{\lambda}\right), \tilde{\rho}\left(y_{\mu},(f, \varphi) y_{\mu}\right),\right. \\
& \left.\frac{\tilde{\rho}\left(y_{\mu},(f, \varphi) x_{\lambda}\right)+\tilde{\rho}\left(y_{\mu},(f, \varphi) y_{\mu}\right)+\tilde{\rho}\left(x_{\lambda},(f, \varphi) x_{\lambda}\right)}{6}\right\} .
\end{aligned}
$$

Hence the condition of corollary (2.9) (theorem 2.7) holds and $(f, \varphi)$ has a fixed point in $A_{1} \cap A_{2}$. Here $x_{\lambda}=0$ is a fixed point of $(f, \varphi)$.

Example 2.11: Let $X=R$ with soft dislocated metric $\tilde{\rho}\left(x_{\lambda}, y_{\mu}\right)=\max \left\{\left|x_{\lambda}\right|,\left|y_{\mu}\right|\right\}$, for all $x_{\lambda}, y_{\mu} \in X$. Suppose $A_{1}=[-1,0]$ and $A_{2}=[0,1] \& Y=\bigcup_{i=1}^{i=2} A_{i}$ we define $(f, \varphi): Y \rightarrow Y$ by

$$
(f, \varphi)= \begin{cases}\frac{\left(x_{\lambda}\right)^{2}}{2}, & \text { if } x_{\lambda} \in[-1,0], \\ \frac{-x_{\lambda}}{4}, & \text { if } x_{\lambda} \in[0,1] .\end{cases}
$$

It is clear that $\bigcup_{i=1}^{i=2} A_{i}$ is a cyclic representation of $Y$ with respect to $(f, \varphi)$. Let $x_{\lambda} \in A_{1}=[-1,0]$ and $y_{\mu} \in A_{2}=[0,1]$, then

$$
\begin{aligned}
& \tilde{\rho}\left((f, \varphi) x_{\lambda},(f, \varphi) y_{\mu}\right) \\
= & \tilde{\rho}\left(\frac{x_{\lambda}^{2}}{2}, \frac{-y_{\mu}}{2}\right)=\max \left\{\left|\frac{x_{\lambda}^{2}}{2}\right|,\left|\frac{-y_{\mu}}{4}\right|\right\} \leq \max \left\{\frac{x_{\lambda}}{2}, \frac{-y_{\mu}}{2}\right\} \\
& \leq \frac{1}{2} \max \left\{x_{\lambda},-y_{\mu}\right\} \leq \frac{1}{2} \max \left\{\left|x_{\lambda}\right|,\left|y_{\mu}\right|\right\} \leq \frac{1}{2} \tilde{\rho}\left(x_{\lambda}, y_{\mu}\right), \\
\tilde{\rho} & \left((f, \varphi) x_{\lambda},(f, \varphi) y_{\mu}\right) \\
= & r \max \left\{\tilde{\rho}\left(x_{\lambda}, y_{\mu}\right), \tilde{\rho}\left(x_{\lambda},(f, \varphi) x_{\lambda}\right), \tilde{\rho}\left(y_{\mu},(f, \varphi) y_{\mu}\right),\right. \\
& \left.\frac{\tilde{\rho}\left(y_{\mu},(f, \varphi) x_{\lambda}\right)+\tilde{\rho}\left(y_{\mu},(f, \varphi) y_{\mu}\right)+\tilde{\rho}\left(x_{\lambda},(f, \varphi) x_{\lambda}\right)}{6}\right\} .
\end{aligned}
$$

Hence the condition of corollary (2.9) (theorem 2.7) holds and $(f, \varphi)$ has a soft fixed point in $A_{1} \cap A_{2}$. Here $x_{\lambda}=0$ is a soft fixed point of $(f, \varphi)$.

In the above corollary we take $A_{i}=(\tilde{X}, \tilde{\rho}, E)$ for all $0 \leq i \leq m$, then we deduce the following corollary. 
Corollary 2.12: Let $(\tilde{X}, \tilde{\rho}, E)$ be a complete soft dislocated metric space and let $(f, \varphi)$ be a self map on $X$. Assume that there exist $r \in[0,1)$ such that

$$
\begin{aligned}
& \tilde{\rho}\left((f, \varphi) x_{\lambda},(f, \varphi) y_{\mu}\right) \\
& \leq r \max \left\{\tilde{\rho}\left(x_{\lambda}, y_{\mu}\right), \tilde{\rho}\left(x_{\lambda},(f, \varphi) x_{\lambda}\right), \tilde{\rho}\left(y_{\mu},(f, \varphi) y_{\mu}\right),\right. \\
& \left.\frac{\tilde{\rho}\left(y_{\mu},(f, \varphi) x_{\lambda}\right)+\tilde{\rho}\left(y_{\mu},(f, \varphi) y_{\mu}\right)+\tilde{\rho}\left(x_{\lambda},(f, \varphi) x_{\lambda}\right)}{6}\right\},
\end{aligned}
$$

holds for all $x_{\lambda}, y_{\mu} \in(X, \tilde{\rho}, E)$. Then $(f, \varphi)$ has a soft fixed point. Moreover if $\tilde{\rho}\left(x_{\lambda}, y_{\mu}\right) \geq \tilde{\rho}\left(x_{\lambda}, x_{\lambda}\right)$ for all $x_{\lambda}, y_{\mu} \in \operatorname{Fix}((f, \varphi))$, then $(f, \varphi)$ has a unique soft fixed point.

Example 2.13: Let $X=R$ with soft dislocated metric space and $\tilde{\rho}\left(x_{\lambda}, y_{\mu}\right)=\max \left\{x_{\lambda}, y_{\mu}\right\}$. For any $x_{\lambda}$, let $(f, \varphi):(X, \tilde{\rho}, E) \rightarrow(X, \tilde{\rho}, E)$ be defined by

$$
(f, \varphi) x_{\lambda}= \begin{cases}\frac{x_{\lambda}}{6}, & \text { if } 0 \leq x_{\lambda}<\frac{1}{2} \\ \frac{x_{\lambda}^{2}}{5}, & \text { if } \frac{1}{2} \leq x_{\lambda} \leq 1 \\ \frac{x_{\lambda}}{7}, & \text { if } x>1 .\end{cases}
$$

Proof: To show that the existence and uniqueness of soft point of $(f, \varphi)$, we need to consider the following cases

Let $0 \leq x_{\lambda}, y_{\mu}<\frac{1}{2}$ then

$$
\tilde{\rho}\left((f, \varphi) x_{\lambda},(f, \varphi) y_{\mu}\right)=\frac{1}{6} \max \left\{x_{\lambda}, y_{\mu}\right\} \leq \frac{1}{2} \max \left\{x_{\lambda}, y_{\mu}\right\}=\frac{1}{2} \tilde{\rho}\left(x_{\lambda}, y_{\mu}\right) .
$$

Let $\frac{1}{2} \leq x_{\lambda}, y_{\mu} \leq 1$ then

$$
\begin{aligned}
\tilde{\rho}\left((f, \varphi) x_{\lambda},(f, \varphi) y_{\mu}\right) & =\frac{1}{5} \max \left\{x_{\lambda}^{2}, y_{\mu}^{2}\right\} \leq \frac{1}{5} \max \left\{x_{\lambda}, y_{\mu}\right\} \\
& \leq \frac{1}{2} \max \left\{x_{\lambda}, y_{\mu}\right\}=\frac{1}{2} \tilde{\rho}\left(x_{\lambda}, y_{\mu}\right) .
\end{aligned}
$$

Let $x_{\lambda}, y_{\mu}>1$ then

$$
\tilde{\rho}\left((f, \varphi) x_{\lambda},(f, \varphi) y_{\mu}\right)=\frac{1}{7} \max \left\{x_{\lambda}, y_{\mu}\right\} \leq \frac{1}{2} \max \left\{x_{\lambda}, y_{\mu}\right\}=\frac{1}{2} \tilde{\rho}\left(x_{\lambda}, y_{\mu}\right) .
$$

Let $0 \leq x_{\lambda}<\frac{1}{2}$ and $\frac{1}{2} \leq y_{\mu} \leq 1$ then

$$
\tilde{\rho}\left((f, \varphi) x_{\lambda},(f, \varphi) y_{\mu}\right)=\max \left\{\frac{x_{\lambda}}{6}, \frac{y_{\mu}^{2}}{5}\right\} \leq \frac{1}{2} \max \left\{x_{\lambda}, y_{\mu}\right\}=\frac{1}{2} \tilde{\rho}\left(x_{\lambda}, y_{\mu}\right) \text {. }
$$

Let $0 \leq x_{\lambda}<\frac{1}{2}$ and $y_{\mu}>1$ then

$$
\tilde{\rho}\left((f, \varphi) x_{\lambda},(f, \varphi) y_{\mu}\right)=\max \left\{\frac{x_{\lambda}}{6}, \frac{y_{\mu}}{7}\right\} \leq \frac{1}{2} \max \left\{x_{\lambda}, y_{\mu}\right\}=\frac{1}{2} \tilde{\rho}\left(x_{\lambda}, y_{\mu}\right) \text {. }
$$


Let $\frac{1}{2} \leq x_{\lambda} \leq 1$ and $0 \leq y_{\mu} \leq \frac{1}{2}$ then

$$
\tilde{\rho}\left((f, \varphi) x_{\lambda},(f, \varphi) y_{\mu}\right)=\max \left\{\frac{x_{\lambda}}{5}, \frac{y_{\mu}}{6}\right\} \leq \frac{1}{2} \max \left\{x_{\lambda}, y_{\mu}\right\}=\frac{1}{2} \tilde{\rho}\left(x_{\lambda}, y_{\mu}\right) .
$$

and so

$$
\begin{aligned}
& \tilde{\rho}\left((f, \varphi) x_{\lambda},(f, \varphi) y_{\mu}\right) \\
& \leq r \max \left\{\tilde{\rho}\left(x_{\lambda}, y_{\mu}\right), \tilde{\rho}\left(x_{\lambda},(f, \varphi) x_{\lambda}\right), \tilde{\rho}\left(y_{\mu},(f, \varphi) y_{\mu}\right),\right. \\
& \left.\frac{\tilde{\rho}\left(y_{\mu},(f, \varphi) x_{\lambda}\right)+\tilde{\rho}\left(y_{\mu},(f, \varphi) y_{\mu}\right)+\tilde{\rho}\left(x_{\lambda},(f, \varphi) x_{\lambda}\right)}{6}\right\} .
\end{aligned}
$$

Hence we conclude that all the condition of corollary (2.12) (theorem 2.7) holds and $(f, \varphi)$ has a soft fixed point in $[0, \infty)$. By corollary 2.9 we deduce the following result.

Corollary 2.14: Let $(\tilde{X}, \tilde{\rho}, E)$ be a complete soft dislocated metric, $m \in N$, let $A_{1}, A_{2}, \cdots, A_{m}$ be non empty $\tilde{\rho}$-closed subsets of $X$ and $Y=\bigcup_{i=1}^{i=m} A_{i}$. Suppose that $(f, \varphi): Y \rightarrow Y$ is an operator such that

i. $\quad Y=\bigcup_{i=1}^{i=m} A_{i}$ is a cyclic representation of $(\tilde{X}, \tilde{\rho}, E)$ with respect to $(f, \varphi)$.

ii. there exist $r \in[0,1)$ such that

$$
\begin{gathered}
\int_{0}^{\tilde{\rho}\left((f, \varphi) x_{\lambda},(f, \varphi) y_{\mu}\right)} \rho(t) \mathrm{d} t \leq r \int_{0}^{m} \rho(t) \mathrm{d} t, \\
m=\max \left\{\tilde{\rho}\left(x_{\lambda}, y_{\mu}\right), \tilde{\rho}\left(x_{\lambda},(f, \varphi) x_{\lambda}\right), \tilde{\rho}\left(y_{\mu},(f, \varphi) y_{\mu}\right),\right.
\end{gathered}
$$

$$
\left.\frac{\tilde{\rho}\left(y_{\mu},(f, \varphi) x_{\lambda}\right)+\tilde{\rho}\left(y_{\mu},(f, \varphi) y_{\mu}\right)+\tilde{\rho}\left(x_{\lambda},(f, \varphi) x_{\lambda}\right)}{6}\right\},
$$

for any $x \in A_{i}$

$y \in A_{i+1}, i=1,2,3, \cdots, m$, where $A_{m+1}=A_{1}$ and $\rho:[0, \infty) \rightarrow[0, \infty)$ be Lebesgueintegrable mapping satisfying $\int_{0}^{\epsilon} \rho(t) \mathrm{d} t>0$, for $\epsilon>0$. Then $(f, \varphi)$ has a fixed point $z_{\omega} \in \bigcap_{i=1}^{m} A_{i}$. Moreover if $\tilde{\rho}\left(x_{\lambda}, y_{\mu}\right) \geq \tilde{\rho}\left(x_{\lambda}, x_{\lambda}\right)$ for all $x_{\lambda}, y_{\mu} \in \operatorname{Fix}((f, \varphi))$, then $(f, \varphi)$ has a unique soft fixed point.

Definition 2.15: Let $(f, \varphi):(X, \tilde{\rho}, E) \rightarrow(X, \tilde{\rho}, E)$ with $\psi:(X, \tilde{\rho}, E) \rightarrow[0, \infty)$ and $\gamma \in[0,1]$. A mapping $(f, \varphi)$ is said to be a $\gamma-\psi$ sub admissible soft mapping if $\psi\left(x_{\lambda}\right) \leq \gamma \Rightarrow \psi\left((f, \varphi) x_{\lambda}\right) \leq \gamma$, for $x_{\lambda} \in(X, \tilde{\rho}, E)$.

Example 2.16: Let $(f, \varphi):[-\pi, \pi] \rightarrow[-\pi, \pi]$ and $\psi:[-\pi, \pi] \rightarrow R_{+}$be defined by $(f, \varphi) x_{\lambda}=\frac{\pi}{4}\left(\tan x_{\lambda}\right)$ and $\psi\left(x_{\lambda}\right)=\left|x_{\lambda}-\frac{1}{4} \pi\right|+\frac{1}{2}$ then $(f, \varphi)$ is a $\gamma-\psi$ sub admissible mapping, where $\gamma=\frac{1}{2}$, indeed if $\psi\left(x_{\lambda}\right)=\left|x_{\lambda}-\frac{1}{4} \pi\right|+\frac{1}{2} \leq \frac{1}{2}$ then $x_{\lambda}=\frac{1}{4} \pi$.

Hence $(f, \varphi)\left(x_{\lambda}\right)=\frac{1}{4} \pi$ and $\psi\left((f, \varphi) x_{\lambda}\right)=\frac{1}{2}$. 
Let $\Lambda$ be the class of all the functions $\phi:[0, \infty)^{3} \rightarrow[0, \infty)$ that are a continuous with the property: $\phi(x, y, z)=0$ if and only if $x=y=z=0$.

Definition 2.17: Let $(X, \tilde{\rho}, E)$ be a soft dislocated metric space, $m \in N$, let $A_{1}, A_{2}, \cdots, A_{m}$ be $\tilde{\rho}$-closed non empty subsets of $(X, \tilde{\rho}, E)$ and let $Y=\bigcup_{i=1}^{i=m} A_{i}$. Assume that $(f, \varphi): Y \rightarrow Y$ is $\gamma$ - $\psi$-sub admissible mapping, where $\gamma=\frac{1}{8}$.

Then $(f, \varphi)$ is called $\psi$-cyclic generalized weakly C-contraction if

i. $Y=\bigcup_{i=1}^{i=m} A_{i}$ is a cyclic representation of $Y$ with respect to $(f, \varphi)$,

$$
\begin{aligned}
& \tilde{\rho}\left((f, \varphi) x_{\lambda},(f, \varphi) y_{\mu}\right) \\
& \leq \psi\left(x_{\lambda}\right) \tilde{\rho}\left(y_{\mu},(f, \varphi) x_{\lambda}\right)+\psi\left((f, \varphi) x_{\lambda}\right) \tilde{\rho}\left(x_{\lambda},(f, \varphi) y_{\mu}\right) \\
& +\psi\left((f, \varphi)^{2} x_{\lambda}\right) \tilde{\rho}\left(y_{\mu},(f, \varphi) y_{\mu}\right)+\psi\left((f, \varphi)^{3} x_{\lambda}\right) \tilde{\rho}\left(x_{\lambda},(f, \varphi) x_{\lambda}\right) \\
& +\psi\left((f, \varphi)^{4} x_{\lambda}\right) \frac{\tilde{\rho}\left(x_{\lambda},(f, \varphi) x_{\lambda}\right) \tilde{\rho}\left(y_{\mu},(f, \varphi) y_{\mu}\right)}{1+\tilde{\rho}\left(x_{\lambda}, y_{\mu}\right)} \\
& +\psi\left((f, \varphi)^{5} x_{\lambda}\right) \frac{\tilde{\rho}\left(x_{\lambda},(f, \varphi) x_{\lambda}\right) \tilde{\rho}\left(x_{\lambda},(f, \varphi) y_{\mu}\right)}{1+\tilde{\rho}\left(y_{\mu},(f, \varphi) x_{\lambda}\right)} \\
& -\phi\left\{\tilde{\rho}\left(x_{\lambda},(f, \varphi) x_{\lambda}\right), \tilde{\rho}\left(y_{\mu},(f, \varphi) y_{\mu}\right)\right. \\
& \left.\frac{1}{2}\left[\tilde{\rho}\left(x_{\lambda},(f, \varphi) y_{\mu}\right)+\tilde{\rho}\left(y_{\mu},(f, \varphi) x_{\lambda}\right)\right]\right\}
\end{aligned}
$$

for any $x \in A_{i}, \quad y \in A_{i+1}, \quad i=1,2,3, \cdots, m$ and $A_{m+1}=A_{1}$ and $\phi \in \Lambda$.

Theorem 1.18: Let $(X, \tilde{\rho}, E)$ be a complete soft dislocated metric space, $m \in N$, let $A_{1}, A_{2}, \cdots, A_{m}$, be $\tilde{\rho}$-closed non-empty subsets of $(X, \tilde{\rho}, E)$ and let $Y=\bigcup_{i=1}^{i=m} A_{i}$. Assume that $(f, \varphi): Y \rightarrow Y$ is a $\psi$-cyclic generalized weakly Ccontraction. If there exists $x_{\lambda_{0}}^{0} \in Y$ such that $\psi\left(x_{\lambda}^{0}\right) \leq \frac{1}{8}$, then $(f, \varphi)$ has a soft fixed point $z_{\omega} \in \bigcap_{i=1}^{n} A_{i}$. Moreover if $\psi\left(z_{w_{0}}^{0}\right) \leq \frac{1}{8}$, then $z_{w_{0}}^{0}$ is unique.

Proof: Let $x_{\lambda_{0}}^{0} \in Y$ be such that $\psi\left(x_{\lambda_{0}}^{0}\right) \leq \frac{1}{8}$. Since $(f, \varphi)$ is a sub $\psi$-admissible mapping with respect to $\frac{1}{8}$, then $\psi\left((f, \varphi) x_{\lambda_{0}}^{0}\right) \leq \frac{1}{8}$.

$\psi\left((f, \varphi)^{n} x_{\lambda_{0}}^{0}\right) \leq \frac{1}{8}$ for all $n \in N \cup 0$. Also, there exist some $\mathrm{i}_{0}$ such that $x_{\lambda_{0}}^{0} \in A_{i_{0}}$. Now $(f, \varphi)\left(A_{i_{0}}\right) \subseteq A_{i_{0}+1}$ implies that $(f, \varphi)\left(x_{\lambda_{0}}^{0}\right) \in A_{i_{0}+1}$. Thus there exist $x_{\lambda_{1}}^{1}$ in $A_{i_{0}+1}$ such that $(f, \varphi) x_{\lambda_{0}}^{0}=x_{\lambda_{1}}^{1}$. Similarly $(f, \varphi) x_{\lambda_{n}}^{n}=x_{\lambda_{n+1}}^{n+1}$, where $x_{\lambda_{n}}^{n} \in A_{i_{n}}$. Hence for $n \geq 0$ there exist $i_{n} \in\{1,2,3, \cdots, m\}$ such that $x_{\lambda_{n}}^{n} \in A_{i_{n}}$ and $x_{\lambda_{n+1}}^{n+1} \in A_{i_{n}+1}$. In case $x_{\lambda_{n_{0}}}^{n_{0}}=x_{\lambda_{n_{0}+1}}^{n_{0}+1}$, for some $n_{0}=0,1,2, \cdots$, then it is clear that $x_{\lambda_{n_{0}}}^{n_{0}}$ is a fixed point of $(f, \varphi)$. Now assume that $x_{\lambda_{n}}^{n} \neq x_{\lambda_{n+1}}^{n+1}$, for all $n$.

Since $(f, \varphi): Y \rightarrow Y$ is a cyclic generalized weak C-contraction, we have for all $n \in N^{*}$ we have 


$$
\begin{aligned}
& \tilde{\rho}\left(x_{\lambda_{n}}^{n}, x_{\lambda_{n+1}}^{n+1}\right)=\tilde{\rho}\left((f, \varphi) x_{\lambda_{n-1}}^{n-1},(f, \varphi) x_{\lambda_{n}}^{n}\right) \\
& \leq \psi\left(x_{\lambda_{n-1}}^{n-1}\right) \tilde{\rho}\left(x_{\lambda_{n}}^{n},(f, \varphi) x_{\lambda_{n-1}^{n-1}}^{n-1}\right)+\psi\left((f, \varphi) x_{\lambda_{n-1}^{n-1}}^{n-1}\right) \tilde{\rho}\left(x_{\lambda_{n-1}^{n-1}}^{n-1},(f, \varphi) x_{\lambda_{n}}^{n}\right) \\
& +\psi\left((f, \varphi)^{2} x_{\lambda_{n-1}}^{n-1}\right) \tilde{\rho}\left(x_{\lambda_{n}}^{n},(f, \varphi) x_{\lambda_{n}}^{n}\right)+\psi\left((f, \varphi)^{3} x_{\lambda_{n-1}}^{n-1}\right) \tilde{\rho}\left(x_{\lambda_{n-1}}^{n-1},(f, \varphi) x_{\lambda_{n-1}}^{n-1}\right) \\
& +\psi\left((f, \varphi)^{4} x_{\lambda_{n-1}}^{n-1}\right) \frac{\tilde{\rho}\left(x_{\lambda_{n-1}}^{n-1},(f, \varphi) x_{\lambda_{n-1}^{n-1}}^{n-1}\right) \tilde{\rho}\left(x_{\lambda_{n}}^{n},(f, \varphi) x_{\lambda_{n}}^{n}\right)}{1+\tilde{\rho}\left(x_{\lambda_{n-1}}^{n-1}, x_{\lambda_{n}}^{n}\right)} \\
& +\psi\left((f, \varphi)^{5} x_{\lambda_{n-1}^{n-1}}^{n-1}\right) \frac{\tilde{\rho}\left(x_{\lambda_{n-1}^{n-1}}^{n-1},(f, \varphi) x_{\lambda_{n-1}^{n-1}}^{n-1}\right) \tilde{\rho}\left(x_{\lambda_{n-1}^{n-1}}^{n-1},(f, \varphi) x_{\lambda_{n}}^{n}\right)}{1+\tilde{\rho}\left(x_{\lambda_{n}}^{n},(f, \varphi) x_{\lambda_{n-1}}^{n-1}\right)} \\
& -\phi\left\{\tilde{\rho}\left(x_{\lambda_{n}}^{n},(f, \varphi) x_{\lambda_{n}}^{n}\right), \tilde{\rho}\left(x_{\lambda_{n-1}}^{n-1},(f, \varphi) x_{\lambda_{n-1}}^{n-1}\right)\right. \text {, } \\
& \left.\frac{1}{2}\left[\tilde{\rho}\left(x_{\lambda_{n-1}}^{n-1},(f, \varphi) x_{\lambda_{n}}^{n}\right)+\tilde{\rho}\left(x_{\lambda_{n}}^{n},(f, \varphi) x_{\lambda_{n-1}}^{n-1}\right)\right]\right\} \\
& \leq \frac{1}{8}\left\{\tilde{\rho}\left(x_{\lambda_{n}}^{n}, x_{\lambda_{n}}^{n}\right)+\tilde{\rho}\left(x_{\lambda_{n-1}}^{n-1}, x_{\lambda_{n+1}}^{n+1}\right)+\tilde{\rho}\left(x_{\lambda_{n}}^{n}, x_{\lambda_{n+1}}^{n+1}\right)+\tilde{\rho}\left(x_{\lambda_{n-1}}^{n-1}, x_{\lambda_{n}}^{n}\right)\right. \\
& \left.+\frac{\tilde{\rho}\left(x_{\lambda_{n-1}}^{n-1}, x_{\lambda_{n}}^{n}\right) \tilde{\rho}\left(x_{\lambda_{n}}^{n}, x_{\lambda_{n+1}}^{n+1}\right)}{1+\tilde{\rho}\left(x_{\lambda_{n-1}}^{n-1}, x_{\lambda_{n}}^{n}\right)}+\frac{\tilde{\rho}\left(x_{\lambda_{n-1}}^{n-1}, x_{\lambda_{n}}^{n}\right) \tilde{\rho}\left(x_{\lambda_{n-1}^{n-1}}^{n-1}, x_{\lambda_{n+1}}^{n+1}\right)}{1+\tilde{\rho}\left(x_{\lambda_{n}}^{n}, x_{\lambda_{n}}^{n}\right)}\right\} \\
& -\phi\left\{\tilde{\rho}\left(x_{\lambda_{n}}^{n}, x_{\lambda_{n+1}}^{n+1}\right), \tilde{\rho}\left(x_{\lambda_{n-1}^{n-1}}^{n-1} x_{\lambda_{n}}^{n}\right), \frac{1}{2}\left[\tilde{\rho}\left(x_{\lambda_{n-1}}^{n-1}, x_{\lambda_{n+1}}^{n+1}\right)+\tilde{\rho}\left(x_{\lambda_{n}}^{n}, x_{\lambda_{n}}^{n}\right)\right]\right\} \text {, }
\end{aligned}
$$

And so

$$
\begin{aligned}
& \tilde{\rho}\left(x_{\lambda_{n}}^{n}, x_{\lambda_{n+1}}^{n+1}\right) \\
& \leq \frac{1}{8}\left\{\tilde{\rho}\left(x_{\lambda_{n}}^{n}, x_{\lambda_{n}}^{n}\right)+\tilde{\rho}\left(x_{\lambda_{n-1}^{n-1}}^{n-1} x_{\lambda_{n+1}^{n+1}}^{n+1}\right)+\tilde{\rho}\left(x_{\lambda_{n}}^{n}, x_{\lambda_{n+1}}^{n+1}\right)+\tilde{\rho}\left(x_{\lambda_{n-1}}^{n-1}, x_{\lambda_{n}}^{n}\right)\right. \\
& \left.+\tilde{\rho}\left(x_{\lambda_{n}}^{n}, x_{\lambda_{n+1}}^{n+1}\right)+\frac{\tilde{\rho}\left(x_{\lambda_{n-1}}^{n-1}, x_{\lambda_{n}}^{n}\right) \tilde{\rho}\left(x_{\lambda_{n-1}}^{n-1}, x_{\lambda_{n+1}}^{n+1}\right)}{1+\tilde{\rho}\left(x_{\lambda_{n}}^{n}, x_{\lambda_{n}}^{n}\right)}\right\} .
\end{aligned}
$$

On the other hand from (d3) we have

$$
\tilde{\rho}\left(x_{\lambda_{n-1}}^{n-1}, x_{\lambda_{n+1}}^{n+1}\right) \leq \tilde{\rho}\left(x_{\lambda_{n-1}^{n-1}}^{n-1}, x_{\lambda_{n}}^{n}\right)+\tilde{\rho}\left(x_{\lambda_{n}}^{n}, x_{\lambda_{n+1}}^{n+1}\right) .
$$

And by lemma (2.5D) we have

$$
\tilde{\rho}\left(x_{\lambda_{n}}^{n}, x_{\lambda_{n}}^{n}\right) \leq \tilde{\rho}\left(x_{\lambda_{n-1}}^{n-1}, x_{\lambda_{n}}^{n}\right)+\tilde{\rho}\left(x_{\lambda_{n}}^{n}, x_{\lambda_{n+1}}^{n+1}\right) .
$$

Also

$$
\begin{aligned}
\frac{\tilde{\rho}\left(x_{\lambda_{n-1}}^{n-1}, x_{\lambda_{n}}^{n}\right) \tilde{\rho}\left(x_{\lambda_{n-1}}^{n-1}, x_{\lambda_{n+1}}^{n+1}\right)}{1+\tilde{\rho}\left(x_{\lambda_{n}}^{n}, x_{\lambda_{n}}^{n}\right)} & \leq \frac{\tilde{\rho}\left(x_{\lambda_{n-1}}^{n-1}, x_{\lambda_{n}}^{n}\right) \tilde{\rho}\left(x_{\lambda_{n-1}^{n-1}}^{n-1}, x_{n_{n+1}}^{n+1}\right)}{\tilde{\rho}\left(x_{\lambda_{n}}^{n}, x_{\lambda_{n}}^{n}\right)} \\
& \leq \frac{\tilde{\rho}\left(x_{\lambda_{n-1}}^{n-1}, x_{\lambda_{n}}^{n}\right)\left[\tilde{\rho}\left(x_{\lambda_{n-1}}^{n-1}, x_{\lambda_{n}}^{n}\right)+\tilde{\rho}\left(x_{\lambda_{n}}^{n}, x_{\lambda_{n+1}}^{n+1}\right)\right]}{\tilde{\rho}\left(x_{\lambda_{n-1}}^{n-1}, x_{\lambda_{n}}^{n}\right)+\tilde{\rho}\left(x_{\lambda_{n}}^{n}, x_{\lambda_{n+1}}^{n+1}\right)} \\
& \leq \tilde{\rho}\left(x_{\lambda_{n-1}^{n-1}}^{n-1}, x_{\lambda_{n+1}}^{n+1}\right)
\end{aligned}
$$

From (19) we have 


$$
\begin{aligned}
& \tilde{\rho}\left((f, \varphi) x_{\lambda_{n-1}}^{n-1},(f, \varphi) x_{\lambda_{n}}^{n}\right) \\
\leq & \frac{1}{8}\left\{\tilde{\rho}\left(x_{\lambda_{n-1}}^{n-1}, x_{\lambda_{n}}^{n}\right)+\tilde{\rho}\left(x_{\lambda_{n}}^{n}, x_{\lambda_{n+1}}^{n+1}\right)+\tilde{\rho}\left(x_{\lambda_{n-1}}^{n-1}, x_{\lambda_{n}}^{n}\right)+\tilde{\rho}\left(x_{\lambda_{n}}^{n}, x_{\lambda_{n+1}}^{n+1}\right)\right. \\
& \left.+\tilde{\rho}\left(x_{\lambda_{n}}^{n}, x_{\lambda_{n+1}}^{n+1}\right)+\tilde{\rho}\left(x_{\lambda_{n-1}}^{n-1}, x_{\lambda_{n}}^{n}\right)+\tilde{\rho}\left(x_{\lambda_{n}}^{n}, x_{\lambda_{n+1}}^{n+1}\right)+\tilde{\rho}\left(x_{\lambda_{n-1}}^{n-1}, x_{\lambda_{n}}^{n}\right)\right\} \\
\leq & \frac{1}{8}\left\{4 \tilde{\rho}\left(x_{\lambda_{n-1}}^{n-1}, x_{\lambda_{n}}^{n}\right)+4 \tilde{\rho}\left(x_{\lambda_{n}}^{n}, x_{\lambda_{n+1}}^{n+1}\right)\right\} \\
\leq & \frac{1}{2}\left\{\tilde{\rho}\left(x_{\lambda_{n-1}}^{n-1}, x_{\lambda_{n}}^{n}\right)+\tilde{\rho}\left(x_{\lambda_{n}}^{n}, x_{\lambda_{n+1}}^{n+1}\right)\right\} .
\end{aligned}
$$

Therefore we have

$$
\tilde{\rho}\left(x_{\lambda_{n}}^{n}, x_{\lambda_{n+1}}^{n+1}\right) \leq \tilde{\rho}\left(x_{\lambda_{n-1}}^{n-1}, x_{\lambda_{n}}^{n}\right) .
$$

For any $n \geq 1$, set $t_{n}=\tilde{\rho}\left(x_{\lambda_{n}}^{n}, x_{\lambda_{n-1}}^{n-1}\right)$. On the occasion of the facts above $\left\{t_{n}\right\}$ is a non-increasing sequence of non negative real numbers. Consequently, there exist $L \geq 0$ such that

$$
\lim _{n \rightarrow \infty} \tilde{\rho}\left(x_{\lambda_{n}}^{n}, x_{\lambda_{n-1}}^{n-1}\right)=L .
$$

We shall prove that $L=0$. Since $\tilde{\rho}\left(x_{\lambda_{n}}^{n}, x_{\lambda_{n}}^{n}\right)=2 \phi \tilde{\rho}\left(x_{\lambda_{n}}^{n}, x_{\lambda_{n+1}}^{n+1}\right)$ then we get $\lim _{n \rightarrow \infty} \tilde{d}\left(x_{\lambda_{n}}^{n}, x_{\lambda_{n}}^{n}\right)=2 L$. Similarly, $\lim _{n \rightarrow \infty} \tilde{\rho}\left(x_{\lambda_{n-1}}^{n-1}, x_{\lambda_{n+1}}^{n+1}\right)=2 L$. Then

$$
\lim _{n \rightarrow \infty}\left[\tilde{\rho}\left(x_{\lambda_{n}}^{n}, x_{\lambda_{n}}^{n}\right)+\tilde{\rho}\left(x_{\lambda_{n-1}}^{n-1}, x_{\lambda_{n+1}}^{n+1}\right)\right] \leq 4 L .
$$

On the other hand, by taking limit as $n \rightarrow \infty$ in (19), we have,

$$
L \leq \frac{1}{8}\left[4 L+\left\{\tilde{\rho}\left(x_{\lambda_{n}}^{n}, x_{\lambda_{n}}^{n}\right)+\tilde{\rho}\left(x_{\lambda_{n-1}^{n-1}}^{n-1}, x_{\lambda_{n+1}}^{n+1}\right)\right\}\right],
$$

This implies $4 L \leq \lim _{n \rightarrow \infty}\left\{\tilde{\rho}\left(x_{\lambda_{n}}^{n}, x_{\lambda_{n}}^{n}\right)+\tilde{\rho}\left(x_{\lambda_{n-1}^{n-1}}^{n}, x_{\lambda_{n+1}}^{n+1}\right)\right\}$.

Hence $\lim _{n \rightarrow \infty}\left\{\tilde{\rho}\left(x_{\lambda_{n}}^{n}, x_{\lambda_{n}}^{n}\right)+\tilde{\rho}\left(x_{\lambda_{n-1}}^{n-1}, x_{\lambda_{n+1}}^{n+1}\right)\right\}=4 L$.

Now from (18) we have

$$
\begin{aligned}
& t_{n+1} \leq \psi\left(x_{\lambda_{n-1}}^{n-1}\right) \tilde{\rho}\left(x_{\lambda_{n}}^{n}, x_{\lambda_{n}}^{n}\right)+\psi\left(x_{\lambda_{n}}^{n}\right) \tilde{\rho}\left(x_{\lambda_{n-1}}^{n-1}, x_{\lambda_{n+1}}^{n+1}\right)+\psi\left(x_{\lambda_{n+1}}^{n+1}\right) t_{n+1} \\
&+\psi\left(x_{\lambda_{n+2}}^{n+2}\right) t_{n}+\psi\left(x_{\lambda_{n+3}}^{n+3}\right) t_{n+1}+\psi\left(x_{\lambda_{n+4}}^{n+4}\right) t_{n-1} \frac{\tilde{\rho}\left(x_{\lambda_{n-1}}^{n-1}, x_{\lambda_{n+1}}^{n+1}\right)}{1+\tilde{\rho}\left(x_{\lambda_{n}}^{n}, x_{\lambda_{n}}^{n}\right)} \\
&-\phi\left\{t_{n}, t_{n+1}, \frac{1}{2}\left[\tilde{\rho}\left(x_{\lambda_{n-1}}^{n-1}, x_{\lambda_{n+1}}^{n+1}\right)+\tilde{\rho}\left(x_{\lambda_{n}}^{n}, x_{\lambda_{n}}^{n}\right)\right]\right\} . \\
& t_{n+1} \leq \frac{1}{8}\left\{\tilde{\rho}\left(x_{\lambda_{n}}^{n}, x_{\lambda_{n}}^{n}\right)+\tilde{\rho}\left(x_{\lambda_{n-1}}^{n-1}, x_{\lambda_{n+1}}^{n+1}\right)+t_{n+1}+t_{n}+t_{n+1}+t_{n-1} \frac{\tilde{\rho}\left(x_{\lambda_{n-1}}^{n-1}, x_{\lambda_{n+1}}^{n+1}\right)}{\left.1+\tilde{\rho}\left(x_{\lambda_{n}}^{n}, x_{\lambda_{n}}^{n}\right)\right\}}\right. \\
&-\phi\left\{t_{n}, t_{n+1}, \frac{1}{2}\left[\tilde{\rho}\left(x_{\lambda_{n-1}}^{n-1}, x_{\lambda_{n+1}}^{n+1}\right)+\tilde{\rho}\left(x_{\lambda_{n}}^{n}, x_{\lambda_{n}}^{n}\right)\right]\right\}
\end{aligned}
$$

By taking limit as $n \rightarrow \infty$ in the above inequality, we deduce that, $L \leq L-\phi(L, L, 2 L)$.

So $\phi(L, L, 2 L)=0$, since $\phi\left(x_{\lambda}, y_{\mu}, z_{\omega}\right)=0 \Leftrightarrow x_{\lambda}=y_{\mu}=z_{\omega}=0$, we get $L=0$. 
B. R. Wadkar et al.

Due to $\lim _{n \rightarrow \infty} \tilde{\rho}\left(x_{\lambda_{n}}^{n}, x_{\lambda_{n}}^{n}\right) \leq 2 L$ and $\lim _{n \rightarrow \infty} \tilde{\rho}\left(x_{\lambda_{n-1}}^{n-1}, x_{\lambda_{n+1}}^{n+1}\right) \leq 2 L$ we have

$$
\lim _{n \rightarrow \infty} \tilde{\rho}\left(x_{\lambda_{n}}^{n}, x_{\lambda_{n}}^{n}\right)=\lim _{n \rightarrow \infty} \tilde{\rho}\left(x_{\lambda_{n-1}}^{n-1}, x_{\lambda_{n+1}}^{n+1}\right)=\lim _{n \rightarrow \infty} \tilde{\rho}\left(x_{\lambda_{n}}^{n}, x_{\lambda_{n+1}}^{n+1}\right)=0 .
$$

We shall show that $\left\{x_{\lambda_{n}}^{n}\right\}$ is a $\tilde{\rho}$-Cauchy sequence. At first, we prove the following fact:

(K) for every $\epsilon>0$, there exist $n \in N$ such that if $r, q \geq n$ with $r-q \equiv 1(m)$, then $\tilde{\rho}\left(x_{\lambda_{r}}^{r}, x_{\lambda_{q}}^{q}\right)<\epsilon$.

Suppose to the contrary that there exist $\epsilon>0$, such that for any $n$ in $N$, we can find $r_{n}>q_{n} \geq n$ with $r_{n}-q_{n} \equiv 1(m)$ satisfying

$$
\tilde{\rho}\left(x_{\lambda_{r_{n}}}^{r_{n}}, x_{\lambda_{q_{n}}}^{q_{n}}\right) \geq \epsilon
$$

Following the related lines of the proof of theorem (1.8) we have

$$
\begin{gathered}
\lim _{n \rightarrow \infty} \tilde{\rho}\left(x_{\lambda_{q_{n}}}^{q_{n}}, x_{\lambda_{r_{n}}}^{r_{n}}\right)=\epsilon, \\
\lim _{n \rightarrow \infty} \tilde{\rho}\left(x_{\lambda_{q_{n}+1}}^{q_{n+1}}, x_{\lambda_{r_{n}+1}}^{r_{n}+1}\right)=\epsilon, \\
\lim _{n \rightarrow \infty} \tilde{\rho}\left(x_{\lambda_{q_{n}}}^{q_{n}}, x_{\lambda_{n_{n}+1}}^{r_{n}+1}\right)=\epsilon \text { and } \lim _{n \rightarrow \infty} \tilde{\rho}\left(x_{\lambda_{r_{n}}}^{r_{n}}, x_{\lambda_{q_{n}+1}}^{q_{n}+1}\right)=\epsilon .
\end{gathered}
$$

Since $x_{\lambda_{q_{n}}}^{q_{n}} \& x_{\lambda_{r_{n}}}^{r_{n}}$ lie in different adjacently labeled sets $A_{i}$ and $A_{i+1}$, for a certain $1 \leq i \leq m$. Using the fact that $(f, \varphi)$ is $\psi$-cyclic generalized weakly Ccontraction, we have

$$
\begin{aligned}
& \tilde{\rho}\left(x_{\lambda_{q_{n}+1}}^{q_{n}+1}, x_{\lambda_{r_{n}+1}}^{r_{n}+1}\right)=\tilde{\rho}\left((f, \varphi) x_{\lambda_{q_{n}}}^{q_{n}},(f, \varphi) x_{\lambda_{r_{n}}}^{r_{n}}\right) \\
& \leq \psi\left(x_{\lambda_{q_{n}}}^{q_{n}}\right) \tilde{\rho}\left(x_{\lambda_{r_{n}}}^{r_{n}},(f, \varphi) x_{\lambda_{q_{n}}}^{q_{q_{n}}}\right)+\psi\left((f, \varphi) x_{\lambda_{q_{n}}}^{q_{n}}\right) \tilde{\rho}\left(x_{\lambda_{q_{n}}}^{q_{n}},(f, \varphi) x_{\lambda_{\nu_{n}}}^{r_{n}}\right) \\
& +\psi\left((f, \varphi)^{2} x_{\lambda_{q_{n}}}^{q_{n}}\right) \tilde{\rho}\left(x_{\lambda_{r_{n}}}^{r_{n}},(f, \varphi) x_{\lambda_{r_{n}}}^{r_{n}}\right)+\psi\left((f, \varphi)^{3} x_{\lambda_{q_{n}}}^{q_{n}}\right) \tilde{\rho}\left(x_{\lambda_{q_{n}}}^{q_{n}},(f, \varphi) x_{\lambda_{q_{n}}}^{q_{n}}\right) \\
& +\psi\left((f, \varphi)^{4} x_{\lambda_{q_{n}}}^{q_{n}}\right) \frac{\tilde{\rho}\left(x_{\lambda_{q_{n}}}^{q_{n}},(f, \varphi) x_{\lambda_{q_{n}}}^{q_{n}}\right) \tilde{\rho}\left(x_{\lambda_{r_{n}}}^{r_{n}},(f, \varphi) x_{\lambda_{r_{n}}^{r_{n}}}^{r_{n}}\right)}{1+\tilde{\rho}\left(x_{\lambda_{q_{n}}}^{q_{n}}, x_{\lambda_{r_{n}}}^{r_{n}}\right)} \\
& +\psi\left((f, \varphi)^{5} x_{\lambda_{q_{n}}}^{q_{n}}\right) \frac{\tilde{\rho}\left(x_{\lambda_{q_{n}}}^{q_{n}},(f, \varphi) x_{\lambda_{q_{n}}}^{q_{n}}\right) \tilde{\rho}\left(x_{\lambda_{q_{n}}}^{q_{n}},(f, \varphi) x_{\lambda_{r_{n}}}^{r_{n}}\right)}{1+\tilde{\rho}\left(x_{\lambda_{n_{n}}}^{r_{n}},(f, \varphi) x_{\lambda_{q_{n}}}^{q_{n}}\right)} \\
& -\phi\left\{\tilde{\rho}\left(x_{\lambda_{q_{n}}}^{q_{n}},(f, \varphi) x_{\lambda_{q_{n}}}^{q_{n}}\right), \tilde{\rho}\left(x_{\lambda_{r_{n}}}^{r_{n}},(f, \varphi) x_{\lambda_{r_{n}}}^{r_{n}}\right),\right. \\
& \left.\frac{1}{2}\left[\tilde{\rho}\left(x_{\lambda_{q_{n}}}^{q_{n}},(f, \varphi) x_{\lambda_{r_{n}}}^{r_{n}}\right)+\tilde{\rho}\left(x_{\lambda_{r_{n}}}^{r_{n}},(f, \varphi) x_{\lambda_{q_{n}}}^{q_{n}}\right)\right]\right\} \\
& \leq \frac{1}{8}\left\{\tilde{\rho}\left(x_{\lambda_{r_{n}}}^{r_{n}}, x_{\lambda_{q_{n+1}}}^{q_{n+1}+1}\right)+\tilde{\rho}\left(x_{\lambda_{q_{n}}}^{q_{n}}, \lambda_{\lambda_{r_{n}+1}}^{r_{n+1}}\right)+\tilde{\rho}\left(x_{\lambda_{r_{n}}}^{r_{n}}, x_{\lambda_{r_{n}+1}}^{r_{n+1}}\right)+\tilde{\rho}\left(x_{\lambda_{q_{n}}}^{q_{n}}, x_{\lambda_{q_{n}+1}}^{q_{n+1}}\right)\right. \\
& \left.+\frac{\tilde{\rho}\left(x_{\lambda_{q_{n}}}^{q_{n}}, x_{\lambda_{n_{n}+1}}^{q_{n}+1}\right) \tilde{\rho}\left(x_{\lambda_{r_{n}}}^{r_{n}}, x_{\lambda_{r_{n}+1}}^{r_{n+1}}\right)}{1+\tilde{\rho}\left(x_{\lambda_{q_{n}}}^{q_{n}}, x_{\lambda_{r_{n}}}^{r_{n}}\right)}+\frac{\tilde{\rho}\left(x_{\lambda_{q_{n}}}^{q_{n}}, x_{\lambda_{q_{n+1}}}^{q_{n+1}}\right) \tilde{\rho}\left(x_{\lambda_{q_{n}}}^{q_{n}}, x_{\lambda_{r_{n}+1}}^{r_{n+1}}\right)}{1+\tilde{\rho}\left(x_{\lambda_{r_{n}}}^{r_{n}}, x_{\lambda_{q_{n}+1}}^{q_{n+1}}\right)}\right\}
\end{aligned}
$$

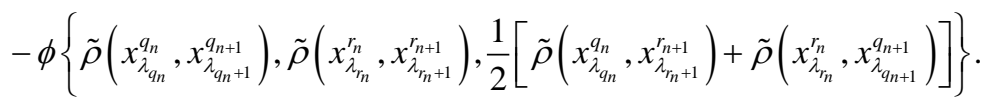

Scientific Research Publishing

125 
Now, by taking limit as $n \rightarrow \infty$ in the above inequality, we derive that

$$
\epsilon \leq \frac{1}{8}[\epsilon+\epsilon+0+0+0+0]-\phi[0,0, \epsilon] \leq \frac{1}{4} \epsilon .
$$

This is a contradiction. Hence condition $(\mathrm{k})$ holds. We are ready to show that the sequence $\left\{x_{\lambda_{n}}^{n}\right\}$ is a Cauchy. Fix $\epsilon>0$. By the claim, we find $n_{0} \in N$ such that if $r, q \geq n_{0}$ with $r-q \equiv 1(m)$ then

$$
\tilde{\rho}\left(x_{\lambda_{r_{n}}}^{r_{n}}, x_{\lambda_{q_{n}}}^{q_{n}}\right) \leq \frac{\epsilon}{4} \leq \frac{\epsilon}{2} .
$$

Since $\lim _{n \rightarrow \infty} \tilde{\rho}\left(x_{\lambda_{n}}^{n}, x_{\lambda_{n+1}}^{n+1}\right)=0$, we find $n_{1} \in N$ such that

$$
\tilde{\rho}\left(x_{\lambda_{n}}^{n}, x_{\lambda_{n+1}}^{n+1}\right) \leq \frac{\epsilon}{2 m},
$$

for any $n \geq n_{1}$. Suppose that $r, s \geq \max \left\{n_{0}, n_{1}\right\}$ with $s>r$. Then there exist $k \in\{1,2, \cdots, m\}$ such that $s-r \equiv k(m)$. Therefore, $s-r+\phi \equiv 1(m)$, for $\phi=m-k+1$.

So we have, for $j \in\{1,2,3, \cdots, m\}, \quad s+j-r \equiv 1(m)$,

$$
\begin{aligned}
\tilde{\rho}\left(x_{\lambda_{r}}^{r}, x_{\lambda_{s}}^{s}\right) \leq & \tilde{\rho}\left(x_{\lambda_{r}}^{r}, x_{\lambda_{s+j}}^{s+j}\right)+\tilde{\rho}\left(x_{\lambda_{r}}^{r}, x_{\lambda_{s+j}}^{s+j}\right) \\
& +\tilde{\rho}\left(x_{\lambda_{s+j}}^{s+j}, x_{\lambda_{s+j-1}}^{s+j-1}\right)+\cdots+\tilde{\rho}\left(x_{\lambda_{s+1}}^{s+1}, x_{\lambda_{s}}^{s}\right)
\end{aligned} .
$$

By (25) and (26) and from the last inequality, we get

$$
\tilde{\rho}\left(x_{\lambda_{r}}^{r}, x_{\lambda_{s}}^{s}\right) \leq \frac{\epsilon}{2}+j \times \frac{\epsilon}{2 m} \leq \frac{\epsilon}{2}+m \times \frac{\epsilon}{2 m}=\epsilon .
$$

This proves that $\left\{x_{\lambda_{n}}^{n}\right\}$ is a $\tilde{\rho}$-Cauchy sequence.

Since $Y$ is $\tilde{\rho}$-closed in $(X, \tilde{\rho}, E)$, then $(Y, \tilde{\rho}, E)$ is also complete, there exists $z_{\omega} \in Y=\bigcup_{i=1}^{m} A_{i}$ such that $\lim _{n \rightarrow \infty} x_{\lambda_{n}}^{n}=z_{\omega}$ in $(Y, \tilde{\rho}, E)$, equivalently

$$
\tilde{\rho}\left(z_{\omega}, z_{\omega}\right)=\lim _{n \rightarrow \infty} \tilde{\rho}\left(z_{\omega}, x_{\lambda_{n}}^{n}\right)=\lim _{m, n \rightarrow \infty} \tilde{\rho}\left(x_{\lambda_{n}}^{n}, x_{\lambda_{m}}^{m}\right)=0 .
$$

In what follows, we prove that $x_{\lambda}$ is a soft fixed point of $(f, \varphi)$. In fact, since $\lim _{n \rightarrow \infty} x_{\lambda_{n}}^{n}=z_{w}$ and as $Y=\bigcup_{i=1}^{m} A_{i}$ is cyclic representation of $Y$ with respect to $(f, \varphi)$, the sequence $\left\{x_{\lambda_{n}}^{n}\right\}$ has infinite terms in each $A_{i}$ for $i \in\{1,2,3, \cdots, m\}$. Suppose that $x_{\lambda} \in A_{i}, \quad(f, \varphi) x_{\lambda} \in A_{i+1}$ and we take a subsequence $\left\{x_{\lambda_{n k}}^{n_{k}}\right\}$ of $\left\{x_{\lambda_{n}}^{n}\right\}$ with $x_{\lambda_{n_{k}}}^{n_{k}} \in A_{i-1}$ (the existence of this subsequence is guaranteed by the above mentioned comment). By using the contractive condition, we can obtain

$$
\begin{aligned}
& \tilde{\rho}\left(x_{\lambda_{n_{k}+1}}^{n_{k}+1},(f, \varphi) x_{\lambda}\right) x_{\lambda_{n_{k}}}^{n_{k}}=\tilde{\rho}\left((f, \varphi) x_{\lambda_{n_{k}}}^{n_{k}},(f, \varphi) x_{\lambda}\right) \\
& \leq \psi\left(x_{\lambda_{n_{k}}}^{n_{k}}\right) \tilde{\rho}\left(x_{\lambda},(f, \varphi) x_{\lambda_{n_{k}}}^{n_{k}}\right)+\psi\left((f, \varphi) x_{\lambda_{n_{k}}}^{n_{k}}\right) \tilde{\rho}\left(x_{\lambda_{n_{k}}}^{n_{k}},(f, \varphi) x_{\lambda}\right) \\
& +\psi\left((f, \varphi)^{2} x_{\lambda_{n_{k}}}^{n_{n_{k}}}\right) \tilde{\rho}\left(x_{\lambda},(f, \varphi) x_{\lambda}\right)+\psi\left((f, \varphi)^{3} x_{\lambda_{n_{k}}}^{n_{k}}\right) \tilde{\rho}\left(x_{\lambda_{n_{k}}}^{n_{k}},(f, \varphi) x_{\lambda_{n_{k}}}^{n_{n_{k}}}\right) \\
& +\psi\left((f, \varphi)^{4} x_{\lambda_{n_{k}}}^{n_{k}}\right) \frac{\tilde{\rho}\left(x_{\lambda_{n_{k}}}^{n_{k}},(f, \varphi) x_{\lambda_{n_{k}}}^{n_{k}}\right) \tilde{\rho}\left(x_{\lambda},(f, \varphi) x_{\lambda}\right)}{1+\tilde{\rho}\left(x_{\lambda_{n_{k}}}^{n_{k}}, x_{\lambda}\right)}
\end{aligned}
$$




$$
\begin{aligned}
+ & \psi\left((f, \varphi)^{5} x_{\lambda_{n_{k}}}^{n_{k}}\right) \frac{\tilde{\rho}\left(x_{\lambda_{n_{k}}}^{n_{n_{k}}},(f, \varphi) x_{\lambda_{n_{k}}}^{n_{k}}\right) \tilde{\rho}\left(x_{\lambda_{n_{k}}}^{n_{n_{k}}},(f, \varphi) x_{\lambda}\right)}{1+\tilde{\rho}\left(x_{\lambda},(f, \varphi) x_{\lambda_{n_{k}}}^{n_{k}}\right)} \\
- & \phi\left\{\tilde{\rho}\left(x_{\lambda_{n_{k}}}^{n_{k}},(f, \varphi) x_{\lambda_{n_{k}}}^{n_{k}}\right), \tilde{\rho}\left(x_{\lambda},(f, \varphi) x_{\lambda}\right),\right. \\
& \left.\frac{1}{2}\left[\tilde{\rho}\left(x_{\lambda_{n_{k}}}^{n_{k}},(f, \varphi) x_{\lambda}\right)+\tilde{\rho}\left(x_{\lambda},(f, \varphi) x_{n_{n_{k}}}^{n_{k}}\right)\right]\right\}, \\
\leq & \frac{1}{8}\left\{\tilde{\rho}\left(x_{\lambda}, x_{\lambda_{n_{k}+1}}^{n_{k}+1}\right)+\tilde{\rho}\left(x_{\lambda_{n_{k}}}^{n_{k}},(f, \varphi) x_{\lambda}\right)+\tilde{\rho}\left(x_{\lambda},(f, \varphi) x_{\lambda}\right)+\tilde{\rho}\left(x_{\lambda_{n_{k}}}^{n_{k}}, x_{\lambda_{n_{k}+1}}^{n_{k}+1}\right)\right. \\
+ & \left.\frac{\tilde{\rho}\left(x_{\lambda_{n_{k}}}^{n_{k}}, x_{\lambda_{n_{k}+1}}^{n_{k}+1}\right) \tilde{\rho}\left(x_{\lambda},(f, \varphi) x_{\lambda}\right)}{1+\tilde{\rho}\left(x_{\lambda_{n_{k}}}^{n_{k}}, x_{\lambda}\right)}+\frac{\tilde{\rho}\left(x_{\lambda_{n_{k}}}^{n_{k}}, x_{\lambda_{n_{k}+1}}^{n_{k}+1}\right) \tilde{\rho}\left(x_{\lambda_{n_{k}}}^{n_{k}},(f, \varphi) x_{\lambda}\right)}{1+\tilde{\rho}\left(x_{\lambda}, x_{n_{n_{k}+1}}^{n_{k}+1}\right)}\right\} \\
- & \phi\left\{\tilde{\rho}\left(x_{\lambda_{n_{k}}}^{n_{k}}, x_{\lambda_{n_{k+1}}}^{n_{k+1}}\right), \tilde{\rho}\left(x_{\lambda},(f, \varphi) x_{\lambda}\right), \frac{1}{2}\left[\tilde{\rho}\left(x_{\lambda_{n_{k}}}^{n_{k}},(f, \varphi) x_{\lambda}\right)+\tilde{\rho}\left(x_{\lambda}, x_{\lambda_{n_{k}+1}}^{n_{k}+1}\right)\right]\right\} .
\end{aligned}
$$

Passing to the limit as $\lim n \rightarrow \infty$ and using $x_{\lambda_{n_{k}}}^{n_{k}} \rightarrow x_{\lambda}$, lower semi-continuity of $\phi$, we have

$$
\begin{aligned}
& \tilde{\rho}\left(x_{\lambda},(f, \varphi) x_{\lambda}\right) \\
\leq & \frac{1}{8}\left\{0+\tilde{\rho}\left(x_{\lambda},(f, \varphi) x_{\lambda}\right)+\tilde{\rho}\left(x_{\lambda},(f, \varphi) x_{\lambda}\right)+0+0+0\right\} \\
& -\phi\left\{0, \tilde{\rho}\left(x_{\lambda},(f, \varphi) x_{\lambda}\right), \frac{1}{2}\left[\tilde{\rho}\left(x_{\lambda},(f, \varphi) x_{\lambda}\right)\right]\right\} \\
\leq & \frac{1}{4} \tilde{\rho}\left(x_{\lambda},(f, \varphi) x_{\lambda}\right) .
\end{aligned}
$$

So $\tilde{\rho}\left(x_{\lambda},(f, \varphi) x_{\lambda}\right)=0$ and therefore $x_{\lambda}$ is fixed point of $(f, \varphi)$.

Finally to prove the uniqueness of soft fixed point theorem, suppose that $y_{\mu}, z_{\omega} \in(X, \tilde{\rho}, E)$ are soft fixed points of $(f, \varphi)$. The cyclic character of $(f, \varphi)$ and the fact that $y_{\mu}, z_{\omega} \in(X, \tilde{\rho}, E)$ are soft fixed points of $(f, \varphi)$ implies that $y_{\mu}, z_{\omega} \in \bigcap_{i=1}^{m} A_{i}$. Also suppose that $\psi\left(y_{\mu}\right) \leq \frac{1}{8}$. By using contractive condition we derive that

$$
\begin{aligned}
& \tilde{\rho}\left(y_{\mu}, z_{\omega}\right)=\tilde{\rho}\left((f, \varphi) y_{\mu},(f, \varphi) z_{\omega}\right) \\
& \leq \psi\left(y_{\mu}\right) \tilde{\rho}\left(z_{\omega},(f, \varphi) y_{\mu}\right)+\psi\left((f, \varphi) y_{\mu}\right) \tilde{\rho}\left(y_{\mu},(f, \varphi) z_{\omega}\right) \\
& +\psi\left((f, \varphi)^{2} y_{\mu}\right) \tilde{\rho}\left(z_{\omega},(f, \varphi) z_{\omega}\right)+\psi\left((f, \varphi)^{3} y_{\mu}\right) \tilde{\rho}\left(y_{\mu},(f, \varphi) y_{\mu}\right) \\
& +\psi\left((f, \varphi)^{4} y_{\mu}\right) \frac{\tilde{\rho}\left(y_{\mu},(f, \varphi) y_{\mu}\right) \tilde{\rho}\left(z_{w},(f, \varphi) z_{w}\right)}{1+\tilde{\rho}\left(y_{\mu}, z_{w}\right)} \\
& +\psi\left((f, \varphi)^{5} y_{\mu}\right) \frac{\tilde{\rho}\left(y_{\mu},(f, \varphi) y_{\mu}\right) \tilde{\rho}\left(y_{\mu},(f, \varphi) z_{w}\right)}{1+\tilde{\rho}\left(z_{w},(f, \varphi) y_{\mu}\right)} \\
& -\phi\left(\tilde{\rho}\left(y_{\mu},(f, \varphi) y_{\mu}\right), \tilde{\rho}\left(z_{w},(f, \varphi) z_{w}\right),\right. \\
& \left.\quad \frac{1}{2}\left[\tilde{\rho}\left(y_{\mu},(f, \varphi) z_{w}\right)+\tilde{\rho}\left(z_{w},(f, \varphi) y_{\mu}\right)\right]\right)
\end{aligned}
$$




$$
\begin{aligned}
\leq & \frac{1}{8}\left\{\tilde{\rho}\left(z_{\omega}, y_{\mu}\right)+\tilde{\rho}\left(y_{\mu}, z_{\omega}\right)+\tilde{\rho}\left(z_{\omega}, z_{\omega}\right)+\tilde{\rho}\left(y_{\mu}, y_{\mu}\right)\right. \\
& \left.+\frac{\tilde{\rho}\left(y_{\mu}, y_{\mu}\right) \tilde{\rho}\left(z_{w}, z_{w}\right)}{1+\tilde{\rho}\left(y_{\mu}, z_{w}\right)}+\frac{\tilde{\rho}\left(y_{\mu}, y_{\mu}\right) \tilde{\rho}\left(y_{\mu}, z_{w}\right)}{1+\tilde{\rho}\left(z_{w}, y_{\mu}\right)}\right\} \\
& -\phi \rho\left(\tilde{\rho}\left(y_{\mu}, y_{\mu}\right), \tilde{\rho}\left(z_{w}, z_{w}\right), \frac{1}{2}\left[\tilde{\rho}\left(y_{\mu}, z_{w}\right)+\tilde{\rho}\left(z_{w}, y_{\mu}\right)\right]\right) \\
\leq & \frac{1}{8}\left\{2 \tilde{\rho}\left(z_{\omega}, y_{\mu}\right)\right\}-\phi\left(0, \tilde{\rho}\left(z_{w}, z_{w}\right), \frac{1}{2}\left[\tilde{\rho}\left(y_{\mu}, z_{w}\right)+\tilde{\rho}\left(z_{w}, y_{\mu}\right)\right]\right) \\
\leq & \frac{1}{4} \tilde{\rho}\left(z_{\omega}, y_{\mu}\right) .
\end{aligned}
$$

This gives us $\tilde{\rho}\left(z_{\omega}, y_{\mu}\right)=0$, that is $z_{\omega}=y_{\mu}$. This finishes the proof.

Corollary 2.19: Let $(X, \tilde{\rho}, E)$ be a complete soft dislocated metric space, $m \in N$, let $A_{1}, A_{2}, \cdots, A_{m}$ be non empty $\tilde{\rho}$-closed subsets of $(X, d, E)$ and let $Y=\bigcup_{i=1}^{i=m} A_{i}$. Suppose that $(f, \varphi): Y \rightarrow Y$ is an operator such that

i) $Y=\bigcup_{i=1}^{i=m} A_{i}$ is cyclic representation of $(X, \tilde{\rho}, E)$ with respect to $(f, \varphi)$;

ii) there exist $\beta \in\left[0, \frac{1}{8}\right)$ such that

$$
\begin{aligned}
& \tilde{\rho}\left((f, \varphi) x_{\lambda},(f, \varphi) y_{\mu}\right) \\
& \leq \beta\left\{\tilde{\rho}\left(y_{\mu},(f, \varphi) x_{\lambda}\right)+\tilde{\rho}\left(x_{\lambda},(f, \varphi) y_{\mu}\right)+\tilde{\rho}\left(y_{\mu},(f, \varphi) y_{\mu}\right)\right. \\
& +\tilde{\rho}\left(x_{\lambda},(f, \varphi) x_{\lambda}\right)+\frac{\tilde{\rho}\left(x_{\lambda},(f, \varphi) x_{\lambda}\right) \tilde{\rho}\left(y_{\mu},(f, \varphi) y_{\mu}\right)}{1+\tilde{\rho}\left(x_{\lambda}, y_{\mu}\right)} \\
& \left.+\frac{\tilde{\rho}\left(x_{\lambda},(f, \varphi) x_{\lambda}\right) \tilde{\rho}\left(x_{\lambda},(f, \varphi) y_{\mu}\right)}{1+\tilde{\rho}\left(y_{\mu},(f, \varphi) x_{\lambda}\right)}\right\},
\end{aligned}
$$

for any $x_{\lambda} \in A_{i}, \quad y_{\mu} \in A_{i+1}, i=1,2,3, \cdots, m$, where $A_{m+1}=A_{1}$. Then $(f, \varphi)$ has fixed point $z_{w} \in \bigcap_{i=1}^{i=m} A_{i}$.

Example 2.20: Let $(X, \tilde{\rho}, E)=R$ with soft dislocates metric space $\tilde{\rho}\left(x_{\lambda}, y_{\mu}\right)=\max \left\{\left|x_{\lambda}\right|,\left|y_{\mu}\right|\right\}$, for any $x_{\lambda}, y_{\mu} \in(X, \tilde{\rho}, E)$, suppose $A_{1}=[-2,0]$ and $A_{2}=[0,2] \& Y=\bigcup_{i=1}^{i=2} A_{i}$, we define $(f, \varphi): Y \rightarrow Y$ by

$$
(f, \varphi) x_{\lambda}= \begin{cases}\frac{-x_{\lambda}}{32}, & \text { if } x_{\lambda} \in[-2,0], \\ \frac{-x_{\lambda}}{16}, & \text { if } x_{\lambda} \in[0,2],\end{cases}
$$

It clear that $\bigcup_{i=1}^{i=2} A_{i}$ is cyclic representation of $Y$ with respect to $(f, \varphi)$.

Proof: Let $x_{\lambda_{1}}^{1} \in A_{1}=[-2,0]$ and $x_{\lambda_{2}}^{2} \in A_{2}=[0,2]$

$$
\begin{aligned}
& \tilde{\rho}\left((f, \varphi) x_{\lambda},(f, \varphi) y_{\mu}\right) \\
& =\max \left\{\left|\frac{-x_{\lambda}}{32}\right|,\left|\frac{-y_{\mu}}{16}\right|\right\} \leq \max \left\{\left|\frac{-x_{\lambda}}{32}\right|, \frac{y_{\mu}}{16}\right\} \leq \max \left\{\frac{-x_{\lambda}}{16}, \frac{y_{\mu}}{16}\right\} \\
& \leq \frac{1}{16} \max \left\{\left|x_{\lambda}\right|,\left|y_{\mu}\right|\right\}=\frac{1}{16} \tilde{\rho}\left(x_{\lambda}, y_{\mu}\right) .
\end{aligned}
$$




$$
\begin{aligned}
& \tilde{\rho}\left((f, \varphi) x_{\lambda},(f, \varphi) y_{\mu}\right) \\
& \leq \frac{1}{16}\left\{\tilde{\rho}\left(y_{\mu},(f, \varphi) x_{\lambda}\right)+\tilde{\rho}\left(x_{\lambda},(f, \varphi) y_{\mu}\right)+\tilde{\rho}\left(y_{\mu},(f, \varphi) y_{\mu}\right)\right. \\
& +\tilde{\rho}\left(x_{\lambda},(f, \varphi) x_{\lambda}\right)+\frac{\tilde{\rho}\left(x_{\lambda},(f, \varphi) x_{\lambda}\right) \tilde{\rho}\left(y_{\mu},(f, \varphi) y_{\mu}\right)}{1+\tilde{\rho}\left(x_{\lambda}, y_{\mu}\right)} \\
& \left.+\frac{\tilde{\rho}\left(x_{\lambda},(f, \varphi) x_{\lambda}\right) \tilde{\rho}\left(x_{\lambda},(f, \varphi) y_{\mu}\right)}{1+\tilde{\rho}\left(y_{\mu},(f, \varphi) x_{\lambda}\right)}\right\} .
\end{aligned}
$$

Hence the condition of corollary (2.19) (theorem 2.18) holds and $(f, \varphi)$ has a soft fixed point in $A_{1} \cap A_{2}$. Here $x_{\lambda}=0$ is a soft fixed point of $(f, \varphi)$.

In the above theorem 2.18, if we take $A_{i}=(X, \tilde{\rho}, E)$, for all $0 \leq i \leq m$ then we deduce the following theorem.

Theorem 2.21: Let $(X, \tilde{\rho}, E)$ be a complete soft dislocated metric space and $(f, \varphi):(X, \tilde{\rho}, E) \rightarrow(X, \tilde{\rho}, E)$ be a sub $\psi$-admissible mapping such that

$$
\begin{aligned}
& \tilde{\rho}\left((f, \varphi) x_{\lambda},(f, \varphi) y_{\mu}\right) \\
& \leq \psi\left(x_{\lambda}\right) \tilde{\rho}\left(y_{\mu},(f, \varphi) x_{\lambda}\right)+\psi\left((f, \varphi) x_{\lambda}\right) \tilde{\rho}\left(x_{\lambda},(f, \varphi) y_{\mu}\right) \\
& +\psi\left((f, \varphi)^{2} x_{\lambda}\right) \tilde{\rho}\left(y_{\mu},(f, \varphi) y_{\mu}\right)+\psi\left((f, \varphi)^{3} x_{\lambda}\right) \tilde{\rho}\left(x_{\lambda},(f, \varphi) x_{\lambda}\right) \\
& +\psi\left((f, \varphi)^{4} x_{\lambda}\right) \frac{\tilde{\rho}\left(x_{\lambda},(f, \varphi) x_{\lambda}\right) \tilde{\rho}\left(y_{\mu},(f, \varphi) y_{\mu}\right)}{1+\tilde{\rho}\left(x_{\lambda}, y_{\mu}\right)} \\
& +\psi\left((f, \varphi)^{5} x_{\lambda}\right) \frac{\tilde{\rho}\left(x_{\lambda},(f, \varphi) x_{\lambda}\right) \tilde{\rho}\left(x_{\lambda},(f, \varphi) y_{\mu}\right)}{1+\tilde{\rho}\left(y_{\mu},(f, \varphi) x_{\lambda}\right)} \\
& -\phi\left\{\tilde{\rho}\left(x_{\lambda},(f, \varphi) x_{\lambda}\right), \tilde{\rho}\left(y_{\mu},(f, \varphi) y_{\mu}\right),\right. \\
& \left.\quad \frac{1}{2}\left[\tilde{\rho}\left(x_{\lambda},(f, \varphi) y_{\mu}\right)+\tilde{\rho}\left(y_{\mu},(f, \varphi) x_{\lambda}\right)\right]\right\} ;
\end{aligned}
$$

for any $x_{\lambda}, y_{\mu} \in(X, \tilde{\rho}, E)$, where $\phi \in \wedge, \psi \in \Psi$. Then $(f, \varphi)$ has unique soft fixed point in $(X, \tilde{\rho}, E)$.

Corollary 2.22: Let $(X, \tilde{\rho}, E)$ be a complete soft dislocated metric space and $(f, \varphi):(X, \tilde{\rho}, E) \rightarrow(X, \tilde{\rho}, E)$ be a sub $\psi$-admissible mapping such that

$$
\begin{aligned}
& \tilde{\rho}\left((f, \varphi) x_{\lambda},(f, \varphi) y_{\mu}\right) \\
& \leq \beta\left\{\tilde{\rho}\left(y_{\mu},(f, \varphi) x_{\lambda}\right)+\tilde{\rho}\left(x_{\lambda},(f, \varphi) y_{\mu}\right)+\tilde{\rho}\left(y_{\mu},(f, \varphi) y_{\mu}\right)\right. \\
& +\tilde{\rho}\left(x_{\lambda},(f, \varphi) x_{\lambda}\right)+\frac{\tilde{\rho}\left(x_{\lambda},(f, \varphi) x_{\lambda}\right) \tilde{\rho}\left(y_{\mu},(f, \varphi) y_{\mu}\right)}{1+\tilde{\rho}\left(x_{\lambda}, y_{\mu}\right)} \\
& \left.+\frac{\tilde{\rho}\left(x_{\lambda},(f, \varphi) x_{\lambda}\right) \tilde{\rho}\left(x_{\lambda},(f, \varphi) y_{\mu}\right)}{1+\tilde{\rho}\left(y_{\mu},(f, \varphi) x_{\lambda}\right)}\right\} ;
\end{aligned}
$$

for any $x_{\lambda}, y_{\mu} \in(X, \tilde{\rho}, E)$, where $\beta \in\left[0, \frac{1}{8}\right)$. Then $(f, \varphi)$ has unique soft fixed point in $(X, \tilde{\rho}, E)$. 
Example 2.23: Let $(X, \tilde{\rho}, E)=R$ with soft dislocated metric space $\tilde{\rho}\left(x_{\lambda}, y_{\mu}\right)=\max \left\{x_{\lambda}, y_{\mu}\right\}$, for any $x_{\lambda}, y_{\mu} \in(X, \tilde{\rho}, E)$. Let $(f, \varphi):(X, \tilde{\rho}, E) \rightarrow(X, \tilde{\rho}, E)$ be defined by

$$
(f, \varphi) x_{\lambda}= \begin{cases}\frac{\left(x_{\lambda}\right)^{2}+x_{\lambda}}{18}, & \text { if } 0 \leq x_{\lambda} \leq 1, \\ \frac{x_{\lambda}}{12}, & \text { if } x_{\lambda} \geq 1,\end{cases}
$$

Proof: To show the existence and uniqueness soft point of $(f, \varphi)$, we investigate the following cases

Let $0 \leq x_{\lambda}, y_{\mu}<1$ then

$$
\begin{aligned}
& \tilde{\rho}\left((f, \varphi) x_{\lambda},(f, \varphi) y_{\mu}\right) \\
& =\max \left\{\frac{x_{\lambda}^{2}+x_{\lambda}}{18}, \frac{y_{\mu}^{2}+y_{\mu}}{18}\right\} \leq \frac{1}{10} \max \left\{x_{\lambda}, y_{\mu}\right\}=\frac{1}{10} \tilde{\rho}\left(x_{\lambda}, y_{\mu}\right) .
\end{aligned}
$$

Let $x_{\lambda}, y_{\mu} \geq 1$ then

$$
\tilde{\rho}\left((f, \varphi) x_{\lambda},(f, \varphi) y_{\mu}\right)=\frac{1}{12} \max \left\{x_{\lambda}, y_{\mu}\right\} \leq \frac{1}{10} \max \left\{x_{\lambda}, y_{\mu}\right\}=\frac{1}{10} \tilde{\rho}\left(x_{\lambda}, y_{\mu}\right)
$$

Let $0 \leq x_{\lambda}<1$ and $y_{\mu} \geq 1$ then

$\tilde{\rho}\left((f, \varphi) x_{\lambda},(f, \varphi) y_{\mu}\right)=\max \left\{\frac{x_{\lambda}^{2}+x_{\lambda}}{18}, \frac{y_{\mu}}{12}\right\} \leq \frac{1}{12} \max \left\{x_{\lambda}, y_{\mu}\right\}=\frac{1}{10} \tilde{\rho}\left(x_{\lambda}, y_{\mu}\right)$.

Hence

$$
\begin{aligned}
& \tilde{\rho}\left((f, \varphi) x_{\lambda},(f, \varphi) y_{\mu}\right) \\
& \leq \frac{1}{10}\left\{\tilde{\rho}\left(y_{\mu},(f, \varphi) x_{\lambda}\right)+\tilde{\rho}\left(x_{\lambda},(f, \varphi) y_{\mu}\right)+\tilde{\rho}\left(y_{\mu},(f, \varphi) y_{\mu}\right)\right. \\
& +\tilde{\rho}\left(x_{\lambda},(f, \varphi) x_{\lambda}\right)+\frac{\tilde{\rho}\left(x_{\lambda},(f, \varphi) x_{\lambda}\right) \tilde{\rho}\left(y_{\mu},(f, \varphi) y_{\mu}\right)}{1+\tilde{\rho}\left(x_{\lambda}, y_{\mu}\right)} \\
& \left.+\frac{\tilde{\rho}\left(x_{\lambda},(f, \varphi) x_{\lambda}\right) \tilde{\rho}\left(x_{\lambda},(f, \varphi) y_{\mu}\right)}{1+\tilde{\rho}\left(y_{\mu},(f, \varphi) x_{\lambda}\right)}\right\} .
\end{aligned}
$$

Hence all the condition of corollary (2.22) (theorem 2.21) are satisfied. Thus $(f, \varphi)$ has a unique soft fixed point in $(X, d, E)$ indeed 0 is the unique soft fixed point of $(f, \varphi)$.

Corollary 2.24: Let $(X, d, E)$ be a complete soft dislocated metric space, $m \in N$, let $A_{1}, A_{2}, \cdots, A_{m}$ be non empty $\tilde{\rho}$-closed subsets of $(X, \tilde{\rho}, E)$ and let $Y=\bigcup_{i=1}^{i=m} A_{i}$. Suppose that $(f, \varphi): Y \rightarrow Y$ is an operator such that

i) $Y=\bigcup_{i=1}^{i=m} A_{i}$ is cyclic representation of $(X, \tilde{\rho}, E)$ with respect to $(f, \varphi)$, ii) $\int_{0}^{\tilde{\rho}\left((f, \varphi) x_{\lambda},(f, \varphi) y_{\mu}\right)} \rho(t) \mathrm{d} t \leq \beta \int_{0}^{m} \rho(t) \mathrm{d} t$.

where

$$
\begin{aligned}
m= & \tilde{\rho}\left(y_{\mu},(f, \varphi) x_{\lambda}\right)+\tilde{\rho}\left(x_{\lambda},(f, \varphi) y_{\mu}\right)+\tilde{\rho}\left(y_{\mu},(f, \varphi) y_{\mu}\right)+\tilde{\rho}\left(x_{\lambda},(f, \varphi) x_{\lambda}\right) \\
& +\frac{\tilde{\rho}\left(x_{\lambda},(f, \varphi) x_{\lambda}\right) \tilde{\rho}\left(y_{\mu},(f, \varphi) y_{\mu}\right)}{1+\tilde{\rho}\left(x_{\lambda}, y_{\mu}\right)}+\frac{\tilde{\rho}\left(x_{\lambda},(f, \varphi) x_{\lambda}\right) \tilde{\rho}\left(x_{\lambda},(f, \varphi) y_{\mu}\right)}{1+\tilde{\rho}\left(y_{\mu},(f, \varphi) x_{\lambda}\right)}
\end{aligned}
$$


for any $x_{\lambda} \in A_{i}, y_{\mu} \in A_{i+1}, i=1,2, \cdots, m$. Where $A_{m+1}=A_{1}$ and $\rho:[0, \infty) \rightarrow[0, \infty)$ is

Lebsegue-integrable mapping satisfying $\int_{0}^{\epsilon} \rho(t) \mathrm{d} t$, for $\epsilon>0$ and the constant $\beta \in\left[0, \frac{1}{8}\right)$. Then $(f, \varphi)$ has unique soft fixed point $z_{\omega} \in \bigcap_{i=1}^{i=m} A_{i}$.

In corollary 2.24, if we take $A_{i}=(X, \tilde{\rho}, E)$, for $i=1,2, \cdots, m$. We obtain the following result.

Theorem 2.25: Let $(X, \tilde{\rho}, E)$ be a complete soft dislocated metric space and $(f, \varphi):(X, \tilde{\rho}, E) \rightarrow(X, \tilde{\rho}, E)$ be a mapping such that for any $x_{\lambda}, y_{\mu} \in(X, \tilde{\rho}, E)$ then

$$
\int_{0}^{\tilde{\rho}\left((f, \varphi) x_{\lambda},(f, \varphi) y_{\mu}\right)} \rho(t) \mathrm{d} t \leq \beta \int_{0}^{m} \rho(t) \mathrm{d} t .
$$

where

$$
\begin{aligned}
m= & \tilde{\rho}\left(y_{\mu},(f, \varphi) x_{\lambda}\right)+\tilde{\rho}\left(x_{\lambda},(f, \varphi) y_{\mu}\right)+\tilde{\rho}\left(y_{\mu},(f, \varphi) y_{\mu}\right)+\tilde{\rho}\left(x_{\lambda},(f, \varphi) x_{\lambda}\right) \\
& +\frac{\tilde{\rho}\left(x_{\lambda},(f, \varphi) x_{\lambda}\right) \tilde{\rho}\left(y_{\mu},(f, \varphi) y_{\mu}\right)}{1+\tilde{\rho}\left(x_{\lambda}, y_{\mu}\right)}+\frac{\tilde{\rho}\left(x_{\lambda},(f, \varphi) x_{\lambda}\right) \tilde{\rho}\left(x_{\lambda},(f, \varphi) y_{\mu}\right)}{1+\tilde{\rho}\left(y_{\mu},(f, \varphi) x_{\lambda}\right)}
\end{aligned}
$$

Let $\rho:[0, \infty) \rightarrow[0, \infty)$ is Lebsegue-integrable mapping satisfying $\int_{0}^{\epsilon} \rho(t) \mathrm{d} t$ for $\epsilon>0$ and the constant $\beta \in\left[0, \frac{1}{8}\right)$. Then $(f, \varphi)$ has unique soft fixed point.

\section{Conclusion}

In this paper, the investigations concerning the existence and uniqueness of soft fixed point of a cyclic mapping in soft dislocated metric space are established. Examples are given in the support of established results. These results can be extended to any directions, and can also be extended to fixed point theory of non-expansive multivalued mappings. These proved results lead to different directions and aspect of soft metric fixed point theory.

\section{Acknowledgements}

The authors would like their sincere thanks to the editor and the anonymous referees for their valuable comments and useful suggestions in improving the article.

\section{References}

[1] Molodtsov, D. (1999) Soft Set-Theory-First Results. Computers \& Mathematics with Applications, 37, 19-31. https://doi.org/10.1016/S0898-1221(99)00056-5

[2] Ali, M.I., Feng, F., Liu, X., Min, W.K. and Shabir, M. (2011) On Some New Operations in Soft Set Theory. Computers \& Mathematics with Applications, 62, 351-358.

[3] Chen, D. (2005) The Parameterization Reduction of Soft Sets and Its Applications. Computers \& Mathematics with Applications, 49, 757-763.

https://doi.org/10.1016/j.camwa.2004.10.036 
[4] Gunduz, C., Sonmez, A. and Çakalli, H. (2013) On Soft Mappings. arXiv:1305.4545v1 [math.GM]

[5] Hussain, S. and Ahmad, B. (2011) Some Properties of Soft Topological Spaces. Computers and Math with Applications, 62, 4058-4067.

https://doi.org/10.1016/j.camwa.2011.09.051

[6] Maji, P.K., Roy, A.R. and Biswas, R. (2002) An Application of Soft Sets in a Decision Making Problem. Computers \& Mathematics with Applications, 44, 1077-1083. https://doi.org/10.1016/S0898-1221(02)00216-X

[7] Maji, P.K., Biswas, R. and Roy, A.R. (2003) Soft Set Theory. Computers and Mathematics with Applications, 45, 555-562. https://doi.org/10.1016/S0898-1221(03)00016-6

[8] Majumdar, P. and Samanta, S.K. (2010) On Soft Mappings. Computers and Mathematics with Applications, 60, 2666-2672. https://doi.org/10.1016/j.camwa.2010.09.004

[9] Shabir, M. and Naz, M. (2011) On Soft Topological Spaces. Computers and Mathematics with Applications, 61, 1786-1799. https://doi.org/10.1016/j.camwa.2011.02.006

[10] Das, S. and Samanta, S.K. (2012) Soft Real Sets, Soft Real Numbers and Their Properties. Journal of Fuzzy Mathematics, 20, 551-576.

[11] Das, S. and Samanta, S.K. (2013) On Soft Metric Spaces. The Journal of Fuzzy Mathematics, 21, 207-213.

[12] Hitzler, P. and Seda, A.K. (2000) Dislocated Topologies. Journal of Electrical Engineering, 51, 3-7.

[13] Aage, C.T. and Salunke, J.N. (2008) Some Results of Fixed Point Theorem in Dislocated Quasi-Metric Spaces. Bulletin of the Marathwada Mathematical Society, 9 , $1-5$.

[14] Karapınar, E. and Salimi, P. (2013) Dislocated Metric Space to Metric Space with Some Fixed Point Theorem. Fixed Point Theory and Application, 2013, 222. https://doi.org/10.1186/1687-1812-2013-222

[15] Bayramov, S. and Gunduz, C. (2013) Soft Locally Compact and Soft Para Compact Spaces. Journal of Mathematics and System Science, 3, 122-130.

[16] Rhoades, B.E. (1977) A Comparison of Various Definitions of Contractive Mappings. Transactions of the American Mathematical Society, 266, 257-290. https://doi.org/10.1090/S0002-9947-1977-0433430-4

[17] Huang, L.-G. and Zhang, X. (2007) Cone Metric Spaces and Fixed Point Theorems of Contractive Mappings. Journal of Mathematical Analysis and Applications, 332, 1468-1476. https://doi.org/10.1016/j.jmaa.2005.03.087

[18] Yazar, M.I., Gunduz, C. and Bayramov, S. (2013) Fixed Point for Soft Contractive Mappings. Cornell University Library, Thurston Ave Ithaca, NY.

[19] Wadkar, B.R., Bhardwaj, R.K. and Singh, B. (2012) A Common Fixed Point Theorem in Dislocated Metric Space. International Journal of Engineering Research and Development, 10, 14-17.

[20] Mishra, L.N., Mishra, V.N., Khatri, K. and Deepmala (2014) On the Trigonometric Approximation of Signals (Functions) Belonging to Generalized Weighted Lipschitz Class by Matrix Operator of Conjugate Series of Its Fourier Series. Applied Mathematics and Computation, 237, 252-263. https://doi.org/10.1016/j.amc.2014.03.085

[21] Mishra, V.N., Khatri, K. and Mishra, L.N. (2013) Using Linear Operator to Approximate Signal of Lip $(\alpha, p),(p \geq 1)$ Class. Filomat, 27, 355-365. 
https://doi.org/10.2298/FIL1302353M

[22] Mishra, L.N., Tiwari, S.K., Mishra, V.N. and Khan, I.A. (2015) Unique Fixed Point Theorems for Generalized Contractive Mappings in Partial Metric Spaces. Journal of Function Spaces, 2015, Article ID: 960827, 8 p. https://doi.org/10.1155/2015/960827

[23] Mishra, L.N., Tiwari, S.K. and Mishra, V.N. (2015) Fixed Point Theorems for Generalized Weakly S-Contractive Mappings in Partial Metric Spaces. Journal of Applied Analysis and Computation, 5, 600-612.

[24] Deepmala and Pathak, H.K. (2013) A Study on Some Problems on Existence of Solutions for Nonlinear Functional-Integral Equations. Acta Mathematica Scientia, 33, 1305-1313. https://doi.org/10.1016/S0252-9602(13)60083-1

[25] Wadkar, B.R., Bhardwaj, R., Mishra, V.N. and Singh, B. (2017) Coupled Fixed Point Theorems in Soft Metric and B-Soft Metric Space. Ser. A: Appl. Math. Inform. And Mech., 9, 59-73. http://www.np.ac.rs/downloads/publications/vol9_br_1/rad6.pdf

[26] Wadkar, B.R., Bhardwaj, R., Singh, B. and Mishra, V.N. (2017) Coupled Fixed Point Theorems with Monotone Property in Soft Metric and Soft B-Metric Space. International Journal of Mathematical Analysis, 11, 363-375.

https://doi.org/10.12988/ijma.2017.7222

Submit or recommend next manuscript to SCIRP and we will provide best service for you:

Accepting pre-submission inquiries through Email, Facebook, LinkedIn, Twitter, etc. A wide selection of journals (inclusive of 9 subjects, more than 200 journals)

Providing 24-hour high-quality service

User-friendly online submission system

Fair and swift peer-review system

Efficient typesetting and proofreading procedure

Display of the result of downloads and visits, as well as the number of cited articles

Maximum dissemination of your research work

Submit your manuscript at: http://papersubmission.scirp.org/

Or contact ojdm@scirp.org 